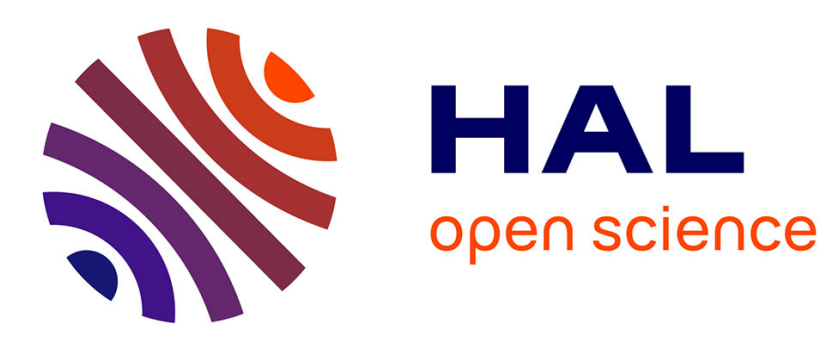

\title{
Coupling damage and cohesive zone models with the Thick Level Set approach to fracture
}

Benoît Lé, Nicolas Moës, Grégory Legrain

\section{To cite this version:}

Benoît Lé, Nicolas Moës, Grégory Legrain. Coupling damage and cohesive zone models with the Thick Level Set approach to fracture. Engineering Fracture Mechanics, 2018, 193, pp.214-247. 10.1016/j.engfracmech.2017.12.036 . hal-02157337

\section{HAL Id: hal-02157337 \\ https://hal.science/hal-02157337}

Submitted on 16 Jun 2019

HAL is a multi-disciplinary open access archive for the deposit and dissemination of scientific research documents, whether they are published or not. The documents may come from teaching and research institutions in France or abroad, or from public or private research centers.
L'archive ouverte pluridisciplinaire HAL, est destinée au dépôt et à la diffusion de documents scientifiques de niveau recherche, publiés ou non, émanant des établissements d'enseignement et de recherche français ou étrangers, des laboratoires publics ou privés. 


\title{
Coupling damage and cohesive zone models with the Thick Level Set approach to fracture
}

\author{
Benoît LÉa,b ${ }^{a, b}$ Nicolas MOËS ${ }^{a, b, *}$, Grégory LEGRAINª,b \\ ${ }^{a}$ Ecole Centrale de Nantes, 1 rue de la Noë, 44321 Nantes, France \\ ${ }^{b}$ GeM Institute, UMR CNRS 6183, 1 rue de la Noë, 44321 Nantes, France
}

\begin{abstract}
This paper couples bulk damage modeling and cohesive zone modeling to get the benefits of both. Damage brings the directionality for the crack propagation as well as the possibility of crack branching while cohesive zone modeling allows for an explicit discrete crack modeling. The coupling is made easy through the Thick Level Set approach. The originality is that the coupling induces concurrent development of bulk and interface degradation.
\end{abstract}

Keywords:

Damage mechanics, Fracture mechanics, Cohesive zone, Thick Level Set, X-FEM, Level Set

\section{Introduction}

Models which allow the study of quasi-brittle failure are usually divided into two categories: continuous and discontinuous models. In the latter, macro-cracks are explicitly modeled by a discontinuity of the displacement field. Cohesive forces $[13,1,17]$ on the crack lips allow to recover the right amount of dissipated energy and process zone length. For continuous models, micro-cracking is modeled by an internal damage variable $[19,7]$, introducing a softening effect on the stiffness of the material. These models do not explicitly represent the displacement discontinuity.

Both continuous and discontinuous models have been widely used to study quasi-brittle materials, therefore their advantages and drawbacks are well-known. Cohesive zone models (CZM) are able to capture macro-cracks openings, which is essential when studying permeability of structures for instance. They are also particularly efficient to represent size effects [18]. However, the discontinuities of the displacement field need to be taken into account by the finite element mesh, which makes crack paths strongly dependent on finite elements orientation. Some particular numerical methods were developed to introduce displacement jumps independently from the spatial discretization, a well-known example being the eXtended Finite Element Method (X-FEM) [27, 26]. Propagation is another complicated aspect, especially in case of branching and coalescence of cracks. Also, an efficient propagation criterion needs to be provided. On the other hand, continuum damage models can easily deal with initiation and complex damage patterns, but can not represent crack opening. Furthermore, local models suffer from spurious mesh dependency [2], which requires some regularization methods. Among these numerous methods we can cite the higher order gradient models $[10,8]$ or regularization of internal variables $[38,16,21,35]$. Based on the variational approach to fracture [14], the phase-field approach [20, 25] uses a smoothed representation of the macro-cracks to get a process zone with a finite thickness, and can deal with complex crack topologies.

Some approaches $[34,45,42,41]$ based on continuum damage mechanics propose to introduce a traction-free discontinuity when the damage variable $D$ becomes greater than a critical value $D_{c}<1$, avoiding conditioning problems when finite elements are fully damaged. However, the amount of energy dissipated when $D$ grows from $D_{c}$ to 1 is not taken into account, and the crack orientation needs to

\footnotetext{
${ }^{*}$ Corresponding author

Email addresses: benoit.le@ec-nantes.fr (Benoît LÉ ), nicolas.moes@ec-nantes.fr (Nicolas MOËS ), gregory.legrain@ec-nantes.fr (Grégory LEGRAIN)
} 


\begin{tabular}{|c|c|}
\hline \multicolumn{2}{|c|}{ Nomenclature } \\
\hline$\ell$ & Position of the damage front (1D case) \\
\hline$\ell_{c}$ & Characteristic length of the TLS \\
\hline$d$ & Interfacial damage \\
\hline$f$ & Traction-separation cohesive function \\
\hline$g$ & Configurational force of the TLSV2 \\
\hline$g_{c}$ & Critical value of the configurational force of the TLSV2 \\
\hline$h$ & Interfacial softening function \\
\hline$h_{e}$ & Mesh element size \\
\hline$k$ & Reference cohesive stiffness \\
\hline$t$ & $1 \mathrm{D}$ cohesive traction \\
\hline$w$ & 1D cohesive opening \\
\hline$w_{c}$ & Critical opening of the cohesive zone model \\
\hline$y$ & Cohesive energy release rate \\
\hline$y_{c}$ & Critical cohesive energy release rate \\
\hline$D$ & Bulk damage \\
\hline$E$ & Young Modulus \\
\hline$H$ & Bulk softening function \\
\hline$Y$ & Bulk energy release rate \\
\hline$Y_{c}$ & Bulk critical energy release rate \\
\hline $\mathbb{C}$ & Order 4 Hooke elasticity tensor \\
\hline$\lambda$ & Augmented Lagrange multiplier \\
\hline$\epsilon$ & $1 \mathrm{D}$ strain \\
\hline$\lambda, \mu$ & Lamé coefficients \\
\hline$\phi$ & Level set field \\
\hline$\phi^{*}$ & Critical value of the TLSV2 \\
\hline$\phi_{s}$ & Value of the level set field on the skeleton \\
\hline$\psi$ & Free energy of the cohesive zone model \\
\hline$\sigma$ & $1 \mathrm{D}$ stress \\
\hline$\sigma_{c}$ & Critical stress of the cohesive zone model \\
\hline$u$ & 1D displacement solution \\
\hline$\Gamma_{s}$ & Skeleton of the level set field \\
\hline$\Psi$ & Free energy of the bulk damage model \\
\hline$(\bullet)_{1}$ & Quantity related to pure CZM or TLSV1 model \\
\hline CZM & Cohesive zone model \\
\hline TLSV1 & Thick Level Set method, first version \\
\hline TLSV2 & Thick Level Set, second version \\
\hline TLS & Thick Level Set \\
\hline$(\bullet)^{\prime}$ & Derivative of the one variable function $(\bullet)$ with respect to its argument \\
\hline$(\bullet)^{n}$ & Quantity $(\bullet)$ computed at computation step $n$ \\
\hline$(\bullet)_{I}$ & Quantity $(\bullet)$ computed at mode $I$ \\
\hline$(\bullet)_{\mathrm{ref}}$ & Quantity $(\bullet)$ computed for the reference loading \\
\hline$[[\bullet]]$ & Jump of $(\bullet)$ field \\
\hline$(\bullet)$ & Increment of $(\bullet)$ \\
\hline$\overline{(\bullet)}$ & Non local TLSV1 field associated to $(\bullet)$ \\
\hline Bold letters & Order 1 or 2 tensors \\
\hline $\operatorname{tr}(\bullet)$ & Trace of tensor $(\bullet)$ \\
\hline
\end{tabular}


be determined. In [15], a different way to proceed is proposed: a traction-free macro-crack is used to compute the displacement solution at a large scale, then the phase field approach is used at the tip scale to propagate this macro-crack. As many multi-scale approaches, the main drawback is that a coupling method between the different scales needs to be provided.

The Thick Level Set method was introduced in [28] and [43] as a new way to regularize local damage models. The implementation was further improved in [3] for quasi-static loading and time-independent damage models, in [31] for dynamics and in [40] for 3D quasi-static problems. The TLS method allows a diffuse progression of damage as well as opening of macro-cracks inside the diffuse zone. It does gather the advantage of both diffuse and strong discontinuity approaches. The use of X-FEM allows to have $D=1$, and therefore to recover the right amount of dissipated energy, while the crack location is given by a level set iso-curve. Note that the TLS approach was compared to the phase-field approach mentioned before in [6].

In previous studies of the TLS, the displacement jump was introduced as a traction-free crack when the material was fully damaged. This version of the TLS will be called TLSV1 in what follows. In this paper, a generalization is proposed, called TLSV2, where we detail the possibility of introducing a displacement jump in a cohesive manner prior to the material being fully damaged. It means that both a cohesive zone and a bulk damaged zone coexist in the TLSV2 model (see figure 1). Equivalence exists between CZM and damage models, see for instance [23, 24] where the behavior of a non-local damage model is shown to converge toward a CZM when the characteristic length tends to zero in 1D, or [5] where a proper way do derive a CZM from a non-local damage model in a thermodynamic framework was established. More recently, a TLS model was determined in [32] in order to have a global behavior similar to a CZM in 1D. However, the TLS regularized damage model and the CZM were not used in a concurrent manner.

Using bulk damage and CZM at the same time allows to overcome some of the difficulties encountered by both of these models. From the CZM point of view:

(a) The bulk damaged zone around the crack will bring the characteristics that are missing to the classical CZM, like propagation criterion and the ability to branch and coalesce.

(b) Another weakness of the CZM is the prediction of cracking in scenarios where stress triaxiality comes into play, for instance in splitting tests of concrete: these models need some improvements to take into account stresses parallel to the crack plane, see for instance [39]. In our approach, the bulk damage around the crack will bring the tensorial information.

From the continuous damage mechanics point of view:

(c) Inserting a cohesive zone before reaching $D=1$ avoids the need to approximate very large strains, while preserving the correct amount of dissipated energy.

(d) It also allows to introduce quite complex interfacial models (like frictional contact between the crack lips), which would be quite tedious to introduce from a bulk modeling point of view.

This paper will demonstrate how the TLSV2 can deal with problems (b) and (c), the other ones will be considered in a forthcoming paper.

Of course, the idea to introduce a cohesive zone inside a damaged zone is not new, see for instance $[9,11,44]$, where a cohesive crack is inserted when the damage variable reaches $D_{c}$. The tractionseparation relation is computed so that the cohesive crack dissipates the energy necessary for the bulk damage to reach 1 , which is more consistent from an energetic point of view compared to the aforementioned approaches where traction-free cracks are introduced.

The originality of the present work is that the TLS permits a concurrent development of dissipation in the bulk and on the interface, with a bulk damage and a cohesive damage coupled by mean of a unique level set field.

The paper is organized as follows. In section 2, the general equations for the TLSV1 damage model, the CZM and the proposed TLSV2 approach are presented on a 1D example. A particular choice for these equations is considered in section 3. Then, the TLSV2 model is extended to 2D and 3D in section 4. Some numerical examples will illustrate the proposed approach in section 5. Finally, a discussion concludes this paper in section 6 . 


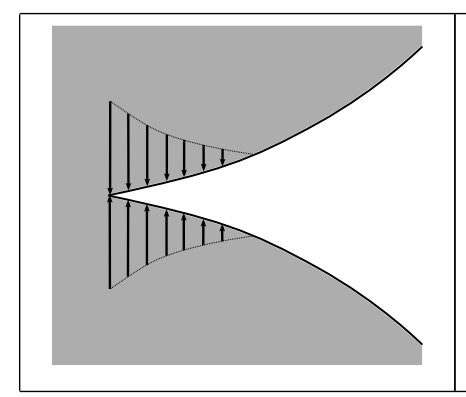

(a) CZM

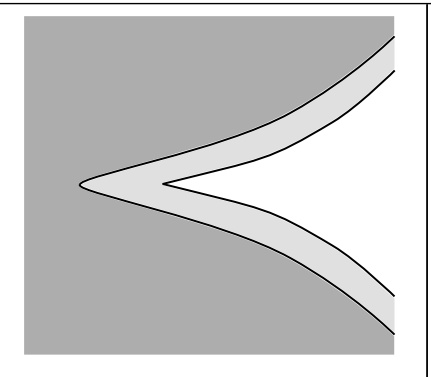

(b) TLSV1

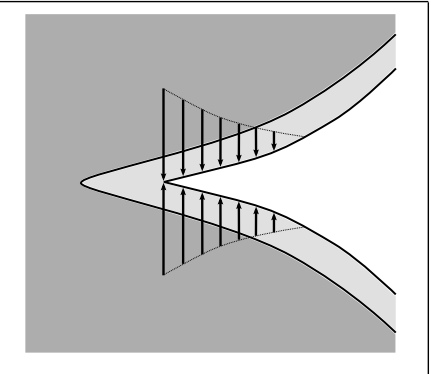

(c) TLSV2

Figure 1: Comparison of the three models: cohesive zone model, TLS damage model (TLSV1), and new version of the TLS model (TLSV2).

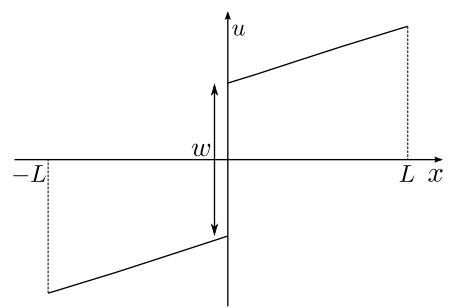

(a)

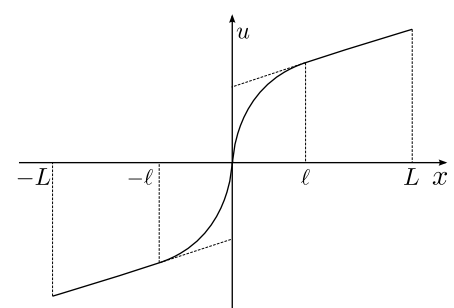

(b)

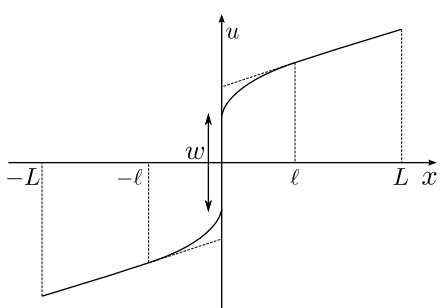

(c)

Figure 2: Displacement $u$ in a 1D bar with strain localization at $x=0$, modeled with three different models: CZM (a), TLSV1 model (b) and TLSV2 model (c).

\section{Model description: $1 \mathrm{D}$ case}

Consider a bar of length $2 L$ and Young modulus $E$ under imposed displacement, leading to tension. Localization is assumed to start at the middle of the bar $(x=0)$. Figure 2 depicts the displacement field along the bar for 3 different models: a pure CZM, a pure TLSV1 damage model and a TLSV2 model, which is a mix between damage model and CZM. These models are detailed in section 2.1, 2.2 and 2.3 respectively. The last model is the target of the paper.

\subsection{The cohesive zone model}

We consider an extrinsic type model (infinite initial stiffness) with an energy given by:

$$
\varphi(w, d)=\frac{1}{2} k\left(\frac{1}{d}-1\right) w^{2}
$$

where $k$ is a reference cohesive stiffness, $d$ the interfacial damage variable and $w$ the displacement jump. It leads to the state equations

$$
t=\frac{\partial \varphi}{\partial w}=k\left(\frac{1}{d}-1\right) w, \quad y=-\frac{\partial \varphi}{\partial d}=\frac{1}{2} k\left(\frac{1}{d}\right)^{2} w^{2}
$$

where $t$ is the cohesive traction and $y$ the cohesive energy release rate. The associated evolution equations are:

$$
\dot{d} \geq 0, \quad y-y_{\mathrm{c}} h(d) \leq 0, \quad\left(y-y_{\mathrm{c}} h(d)\right) \dot{d}=0
$$

where $y_{\mathrm{c}}$ is a constant and $h$ a dimensionless, increasing function such that $h(0)=1$. The model parameters are thus $k, y_{\mathrm{c}}$ and the function $h$.

Usually, a CZM is given as a function $t=f(w)$, with $f(0)=\sigma_{c}$ the tensile strength and $f\left(w_{c}\right)=0$, where $w_{c}$ is the critical opening (see figure 3). It can be shown (see Appendix B) that $y_{\mathrm{c}}$ and $h$ can be determined in order to obtain a model defined by the potential (1) and the evolution equations (3) equivalent to a model defined by a traction-separation function $t=f(w)$. 


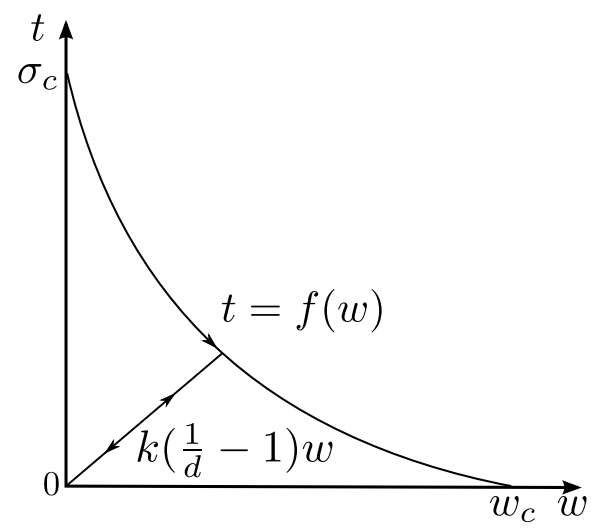

Figure 3: Typical CZM model expressed in terms of a traction-separation function $t=f(w)$, or using the damage variable $d$.

\subsection{TLSV1 approach}

The local damage model is given by the free energy

$$
\Psi(\epsilon, D)=\frac{1}{2}(1-D) E \epsilon^{2}
$$

where $\epsilon$ is the strain in the bar, and $D$ the bulk damage variable. The corresponding state equations are

$$
\sigma=\frac{\partial \Psi}{\partial \epsilon}=(1-D) E \epsilon, \quad Y=-\frac{\partial \Psi}{\partial D}=\frac{1}{2} E \epsilon^{2}
$$

where $\sigma$ is the stress in the bar and $Y$ the bulk energy release rate. The evolution equations are

$$
\dot{D} \geq 0, \quad Y-Y_{\mathrm{c}} H(D) \leq 0 \quad\left(Y-Y_{\mathrm{c}} H(D)\right) \dot{D}=0
$$

where $Y_{\mathrm{c}}$ is a constant and $H$ a dimensionless, increasing function such that $H(0)=1$. The model parameters are thus $E, Y_{\mathrm{c}}$ and the function $H$. This model is purely local, and therefore needs some regularization in order to avoid spurious localization. In the TLS approach a characteristic length $\ell_{\mathrm{c}}$ is introduced, by writing the damage variable as an increasing function $D(\phi)$ of the distance to the damage front (the boundary between the sane and the damaged material) $\phi=\ell-x$, where $\ell$ is the position of the damage front (see figure $2(\mathrm{~b})$ ):

$$
\left\{\begin{array}{r}
D(\phi)=0 \text { if } \quad \phi \leq 0 \\
0<\phi(\phi)<1 \text { if } 0<\phi \leq \ell_{c} \\
D(\phi)=1 \text { if } \ell_{c}<\phi
\end{array}\right.
$$

An example of a parabolic damage profile is given in figure 4. This allows to define non-local quantities:

$$
\bar{Y}=\frac{\int_{0}^{\ell} Y D^{\prime} \mathrm{d} x}{\int_{0}^{\ell} D^{\prime} \mathrm{d} x}, \quad \bar{H}=\frac{\int_{0}^{\ell} H(D) D^{\prime} \mathrm{d} x}{\int_{0}^{\ell} D^{\prime} \mathrm{d} x}, \quad \overline{\dot{D}}=\frac{\int_{0}^{\ell} \dot{D} \mathrm{~d} x}{\int_{0}^{\ell} \mathrm{d} x}
$$

where $D^{\prime}(\phi)$ is the derivative of $D$ with respect to $\phi$, and thefore to obtain the non-local version of the evolution equations (6):

$$
\overline{\dot{D}} \geq 0, \quad \bar{Y}-Y_{\mathrm{c}} \bar{H} \leq 0 \quad\left(\bar{Y}-Y_{\mathrm{c}} \bar{H}\right) \overline{\dot{D}}=0
$$

Note that the definition of $\bar{Y}$ and $\overline{\dot{D}}$ ensures that the local and non-local dissipated energy are equal:

$$
\int_{0}^{\ell} \bar{Y} \overline{\dot{D}} \mathrm{~d} x=\int_{0}^{\ell} Y \dot{D} \mathrm{~d} x
$$




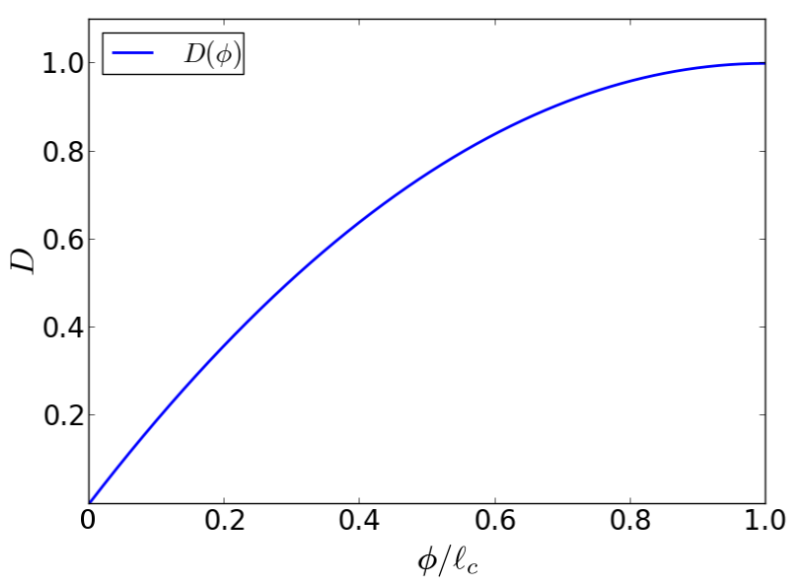

Figure 4: Example of a parabolic damage profile $D(\phi)=2\left(\frac{\phi}{\ell_{c}}\right)-\left(\frac{\phi}{\ell_{c}}\right)^{2}$.

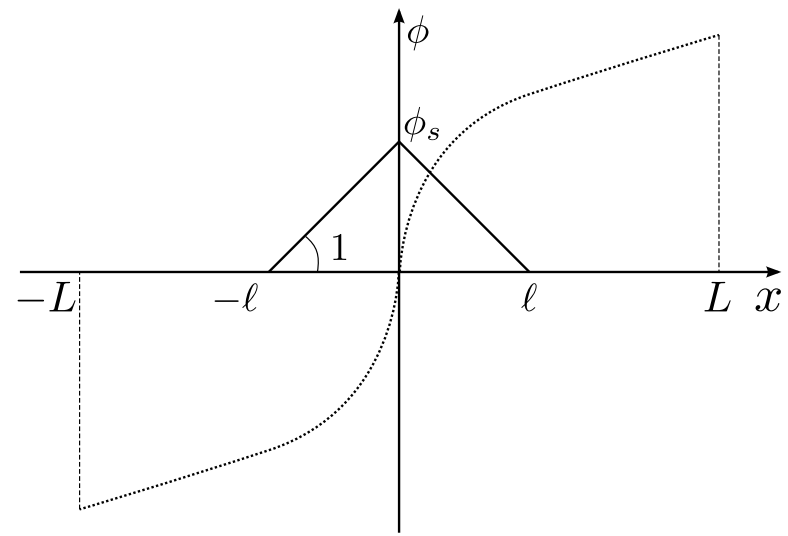

Figure 5: Illustration of a level set field in $1 \mathrm{D}$, with a skeleton located at $x=0$. The displacement field is represented in dotted lines.

Equations (8) and (9) are the equations originally introduced in [28, 43]; they can also be rewritten in an equivalent form which will be more suitable for what follows:

$$
\dot{\phi} \geq 0, \quad z=\int_{0}^{\phi_{s}}\left(Y-Y_{\mathrm{c}} H(D)\right) D^{\prime} \mathrm{d} \phi \leq 0 \quad z \dot{\phi}=0
$$

where $\phi_{s}=\phi\left(x_{s}\right)$, with $x_{s}$ the skeleton of the level set field. As illustrated in figure 5 , this skeleton corresponds to the point where the gradient of the level set field is discontinuous. In this $1 \mathrm{D}$ example it is assumed that $x_{s}=0$. In what follows, the term "skeleton" will always refer to the skeleton of the level set field. The skeleton is a line in 2D and a surface in 3D (see section 4).

\subsection{TLSV2 approach}

At this stage, the above two models are completely independent. The key idea in this paper is to couple them by enforcing that both the damage in the bulk $D$ and the interfacial damage $d$ are functions of $\phi$, as depicted on figure 6. Having $D$ depending on $\phi$ is classical in the TLS framework, the originality here is to have also $d$ depending on $\phi$, and more precisely on $\phi_{s}$. We write $d$ as an increasing function of $\phi_{s}$ :

$$
\left\{\begin{aligned}
d\left(\phi_{s}\right)=0 & \text { if } \\
d\left(\phi_{s}\right)<1 & \text { if } \phi^{*}<\phi_{s} \leq \phi^{*} \leq \ell_{c} \\
d\left(\phi_{s}\right)=1 & \text { if } \ell_{c}<\phi_{s}
\end{aligned}\right.
$$




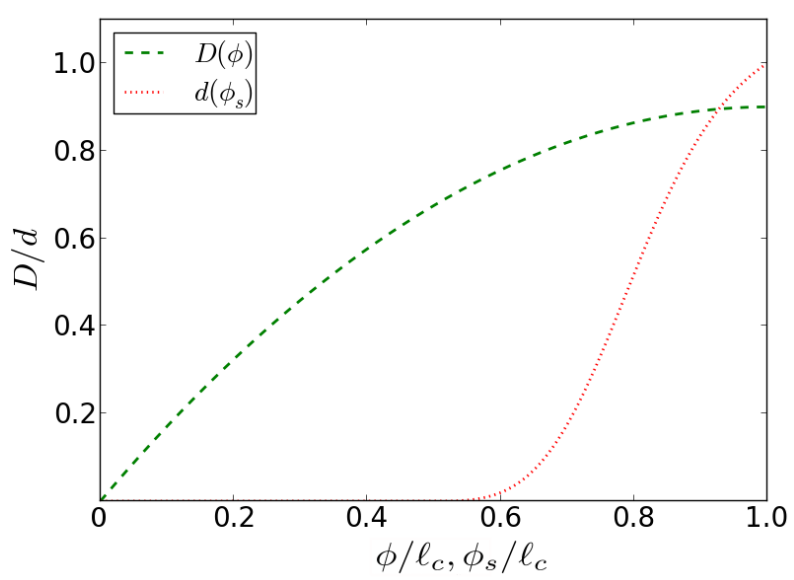

Figure 6: Dependence of the bulk damage $D$ and interfacial damage $d$ with respect to the level set value $\phi$ and the level set value $\phi_{s}=\phi\left(x_{s}\right)$ on the skeleton.

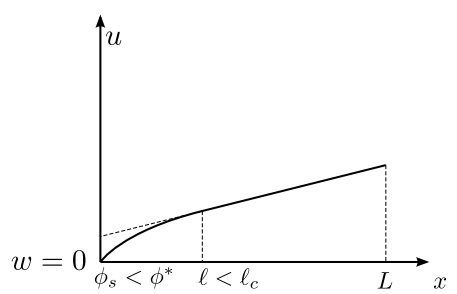

(a)

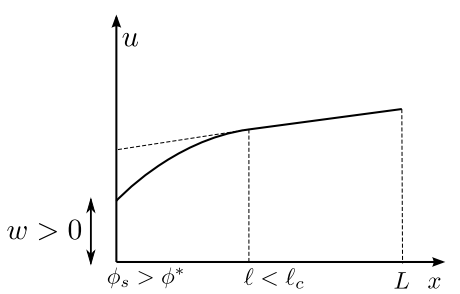

(b)

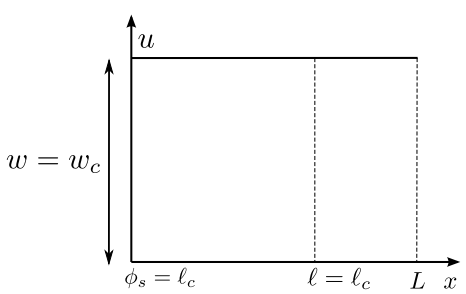

(c)

Figure 7: Displacement solution at different loading stages.

where $\phi^{*}<\ell_{\mathrm{c}}$ is a user defined parameter. The damage profile $D(\phi)$ defined by equation (7) is replaced by a new damage profile $D(\phi)$, increasing function of $\phi_{s}$, such that:

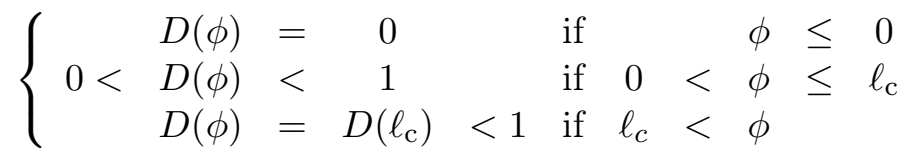

Note that the essential difference with the TLSV1 damage profile defined by equation (7) is that $D(\phi)$ no longer reaches one.

As $\phi$ goes from 0 to $\ell_{\mathrm{c}}, D$ goes from 0 to $D\left(\ell_{\mathrm{c}}\right)<1$ whereas $d$ goes from 0 to 1 as $\phi_{s}$ goes from $\phi^{*}$ to $\ell_{\mathrm{c}}$. Having values of $D(\phi)$ strictly inferior to 1 in the bulk prevents strains from going to infinity. However, some energy still needs to be dissipated to get a fully damaged material; this is done through the evolution of $d$.

Figure 7 depicts the displacement solution for different loading stages:

(a) When $\phi_{s}<\phi^{*}$, the TLSV2 is equivalent to the TLSV1 and there is no displacement jump at $x=0$.

(b) When $\phi_{s}>\phi^{*}$, the interfacial damage variable $d$ starts to grow, and a displacement jump is placed at $x=0$.

(c) $\phi_{s}$ grows until $\phi_{s}=\ell_{\mathrm{c}}$ : the crack faces are traction-free. The displacement jump is equal to the critical opening $w=w_{c}$.

In the TLSV2, two model coexist. However, the evolution of these two models depends only on a single level set field. The two evolution equations (3) and (9) which were driving the evolution of $d$ and $D$ must now be replaced by a single evolution equation driving the evolution of $\phi$. 


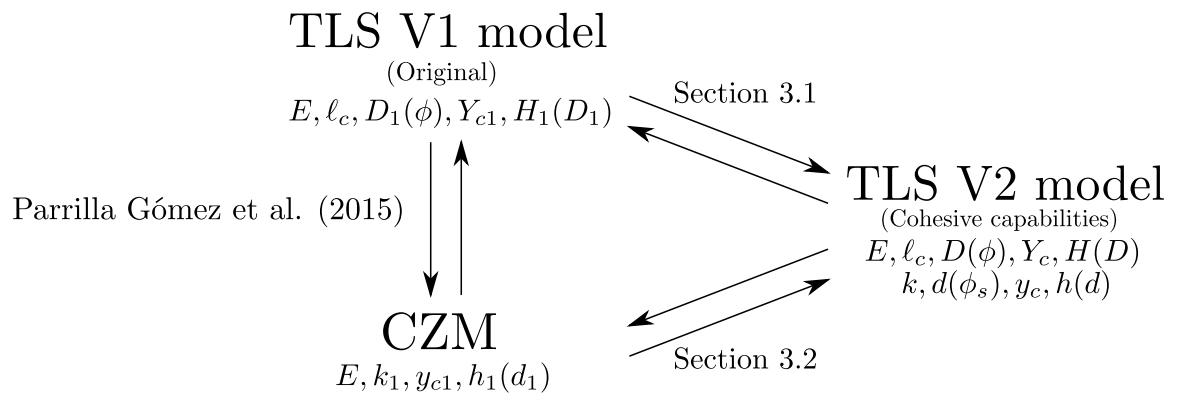

Figure 8: Parameters of the CZM, TLSV1 and TLSV2 models

The configurational force $g$ governing the evolution of $\phi$ on each side of the skeleton is given by

$$
g=\int_{0}^{\phi_{s}} Y D^{\prime} \mathrm{d} \phi+\left.\frac{1}{2} y d^{\prime}\right|_{\phi=\phi_{s}}
$$

where $d^{\prime}$ is the derivative of $d$ with respect to $\phi_{s}$. A half value is needed in the above since the change of the level set value on the skeleton is affected by the average of the level set velocity on each side of the skeleton.

We choose the following expression for the critical value:

$$
g_{\mathrm{c}}=\int_{0}^{\phi_{s}} Y_{\mathrm{c}} H(D) D^{\prime} \mathrm{d} \phi+\left.\frac{1}{2} y_{\mathrm{c}} h(d) d^{\prime}\right|_{\phi=\phi_{s}}
$$

The state equations for the damage and cohesive parts of the model are not modified but we replace the individual evolution equations by the following common one:

$$
\dot{\phi} \geq 0, \quad g-g_{\mathrm{c}} \leq 0, \quad\left(g-g_{\mathrm{c}}\right) \dot{\phi}=0
$$

\section{Relationship between CZM, TLSV1 and TLSV2 models}

The TLSV2 model introduced in the previous section depends on a certain number of parameters, which come from both cohesive and TLSV1 models, as well as the interfacial damage profile $d\left(\phi_{s}\right)$. Regarding notations, all the quantities related to the TLSV1 and CZM models are noted with a subscript "1", whereas the quantities related to TLSV2 are noted without subscript.

In this section, we focus on the relations between these three models, and in particular, how to choose the different parameters in order to have 1D equivalence. This equivalence consists of having the same displacement, energy and dissipation in the 1D example presented in section 2 . The parameters of the different models are summarized in figure 8. For instance, the TLSV2 has 9 parameters that need to be determined. However, as we will see in what follows, only two equations (stiffness and dissipation equivalence) need to be satisfied to achieve the 1D equivalence, which means that 7 of the TLSV2 parameters can be chosen independently. The equivalence between the CZM and the TLSV1 model was established in [32]. In the next sections we will consider the particular cases of the equivalence between TLSV1 and TLSV2, and between CZM and TLSV2. This equivalence is a way, from a known TLSV1 or CZM model, to choose the parameters of the TLSV2. Other ways to choose directly the parameters of the TLSV2 could be foreseen, but have not been investigated in this paper.

For the TLS model, V1 or V2, the damage profile in the bulk is chosen by the user in order to fulfill equation (7) or (13). The characteristic length $\ell_{c}$ will determine the width of the damaged zone: from a numerical point of view, it should be not too small in order to have enough finite elements per $\ell_{\mathrm{c}}$ when computing the integrals in equation (14) and (15). For the TLSV2, the value of $\phi^{*}$ should of course be chosen not too close to $\ell_{\mathrm{c}}$, otherwise the bulk damage $D$ may become close to 1 before the cohesive zone starts to develop. 


\begin{tabular}{|l|l|}
\hline TLSV1 & TLSV2 \\
\hline$E$ & $E$ \\
$\ell_{\mathrm{c}}$ & $\ell_{\mathrm{c}}:$ chosen \\
$D_{1}(\phi)$ & $D(\phi):$ built to fulfill equation $(18)$ \\
$Y_{c 1}$ & $Y_{\mathrm{c}}=Y_{c 1}$ \\
$H_{1}\left(D_{1}\right)$ & $H(D)=H_{1}(D)$ \\
& $k=E /\left(2 \ell_{\mathrm{c}}\right)$ \\
& $d\left(\phi_{s}\right):$ computed from equation $(21)$ \\
& $y_{\mathrm{c}}=2 \ell_{\mathrm{c}} Y_{\mathrm{c}}$ \\
& $h(d):$ computed from equation $(22)$ \\
\hline
\end{tabular}

Table 1: Determination of the TLSV2 parameters from a TLSV1 model.

\subsection{From TLSV1 to TLSV2}

Table 1 summarizes the parameters of the TLSV1 and TLSV2, and how the parameters of the TLSV2 are determined from the TLSV1 parameters.

It is assumed that the TLSV1 model is given: $Y_{c 1}$ and $H_{1}(D)$ are known, and the TLSV1 damage profile is:

$$
D_{1}(\phi)=2\left(\frac{\phi}{\ell_{c}}\right)-\left(\frac{\phi}{\ell_{c}}\right)^{2}
$$

For the TLSV2, the damage profile in the bulk must satisfy:

$$
\left\{\begin{array}{rlrl}
D(\phi) & =D_{1}(\phi) & \text { if } & \phi \leq \phi^{*} \\
D^{*}<1 & <1 & \text { if } \phi^{*}<\phi \leq \ell_{c} \\
D(\phi) & =D\left(\ell_{\mathrm{c}}\right)<1 & \text { if } \ell_{c}<\phi
\end{array}\right.
$$

where $D^{*}=D_{1}\left(\phi^{*}\right)$. The expression of $D(\phi)$ for $\phi>\phi^{*}$ will be derived in Appendix C. Regarding the dissipation parameters $H$ and $Y_{\mathrm{c}}$, we keep the ones coming from the TLSV1 model, namely $H=H_{1}$ and $Y_{\mathrm{c}}=Y_{c 1}$.

Then, the parameters coming from the CZM need to be determined. Values of $k$ and $y_{\mathrm{c}}$ can be chosen, without loss of generality, as:

$$
k=E /\left(2 \ell_{\mathrm{c}}\right)
$$

and

$$
y_{\mathrm{c}}=2 \ell_{\mathrm{c}} Y_{\mathrm{c}}
$$

At the same stress level, both models must have the same energy and dissipation. For $\phi<\phi^{*}$, $d=0, H=H_{1}$ and $D(\phi)=D_{1}(\phi)$, therefore the TLSV1 and TLSV2 are exactly the same. For $\phi>\phi^{*}$, stiffness equivalence implies

$$
2 \int_{0}^{\phi_{s}} \frac{1}{E} \frac{1}{1-D} \mathrm{~d} x+\left.\frac{1}{k} \frac{d}{1-d}\right|_{\phi=\phi_{s}}=2 \int_{0}^{\phi_{s}} \frac{1}{E} \frac{1}{1-D_{1}} \mathrm{~d} x
$$

This relation gives the function $d\left(\phi_{s}\right)$ (see Appendix $\mathrm{C}$ for more details about the determination of $\left.d\left(\phi_{s}\right)\right)$. Note that since $D(\phi)=D_{1}(\phi)$ for $\phi_{s} \leq \phi^{*}, d\left(\phi_{s}\right)=0$ for $\phi_{s} \leq \phi^{*}$, which is in agreement with equation (12). The three damage profiles $D_{1}, D$ and $d$ are plotted on figure 9 .

The next step is to compute $h$. Dissipation equivalence implies:

$$
2 \int_{0}^{\phi_{s}} Y_{\mathrm{c}} H(D) D^{\prime} \mathrm{d} x+\left.y_{\mathrm{c}} h(d) d^{\prime}\right|_{\phi=\phi_{s}}=2 \int_{0}^{\phi_{s}} Y_{\mathrm{c}} H\left(D_{1}\right) D_{1}^{\prime} \mathrm{d} x
$$

which gives the expression of $h$ (see Appendix C for more details about the determination of $h$ ). 


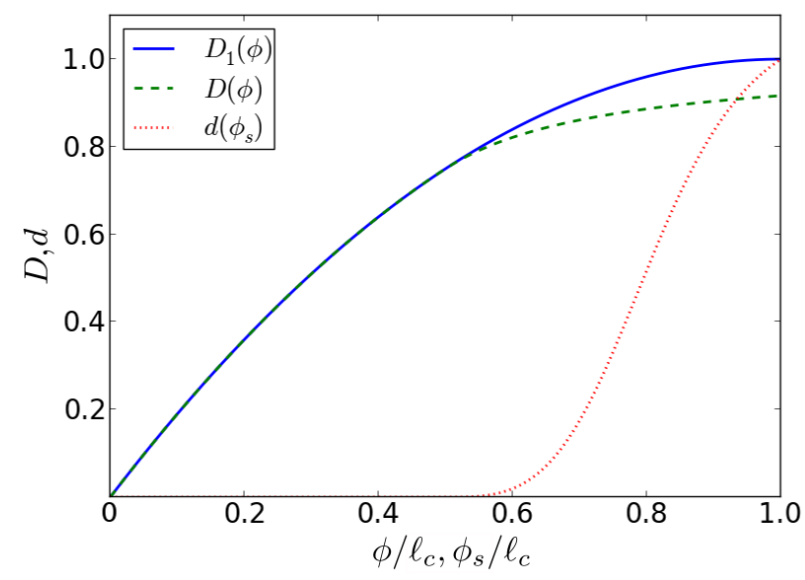

Figure 9: Dependence of the TLSV1 bulk damage $D_{1}(\phi)$, TLSV2 bulk damage $D(\phi)$ and interface damage $d\left(\phi_{s}\right)$ with respect to the level set value in the bulk $\phi$ and on the skeleton $\phi_{s}$, for $\phi^{*}=0.5 \ell_{\mathrm{c}}$. The interface damage $d\left(\phi_{s}\right)$ was obtained from (21).

\begin{tabular}{|l|l|}
\hline CZM & TLSV2 \\
\hline$E$ & $E$ \\
$k_{1}$ & $\ell_{\mathrm{c}}:$ chosen \\
$y_{c 1}$ & $D(\phi):$ chosen \\
$h_{1}\left(d_{1}\right)$ & $Y_{\mathrm{c}}=k_{1}^{-1} E y_{c 1}$ \\
& $H(D):$ computed from equation $(24)$ for $\phi_{s}<\phi^{*}$ \\
& $k=E /\left(2 \ell_{\mathrm{c}}\right)$ \\
& $d\left(\phi_{s}\right):$ chosen to fulfill equation $(12)$ \\
& $y_{\mathrm{c}}=2 \ell_{\mathrm{c}} Y_{\mathrm{c}}$ \\
& $h(d):$ computed from equation $(24)$ for $\phi_{s}>\phi^{*}$ \\
\hline
\end{tabular}

Table 2: Determination of the TLSV2 parameters from a CZM. 


\subsection{From CZM to TLSV2}

Table 2 summarizes the parameters of the CZM and TLSV2, and how the parameters of the TLSV2 are determined from the CZM parameters. We keep for $D, k$ and $y_{\mathrm{c}}$ the same expressions which were used in section 3.1 (equations (18), (19) and (20) respectively). The critical energy release rate $Y_{\mathrm{c}}$ is determined in order to have damage initiation triggered for the same critical stress for which the cohesive crack starts to open in the CZM:

$$
Y_{\mathrm{c}}=k_{1}^{-1} E y_{c 1}
$$

The cohesive damage profile $d\left(\phi_{s}\right)$ is chosen by the user to fulfill equation (12). The remaining parameters to be determined are the functions $H$ and $h$; they are given by the dissipation equivalence:

$$
2 \int_{0}^{\phi_{s}} Y_{\mathrm{c}} H(D) D^{\prime} \mathrm{d} x+\left.y_{\mathrm{c}} h(d) d^{\prime}\right|_{\phi=\phi_{s}}=\left.y_{c 1} h_{1}\left(d_{1}\right) d_{1}^{\prime}\right|_{\phi=\phi_{s}}
$$

where $d_{1}$ is a function of $\phi_{s}$ given by the stiffness equivalence:

$$
2 \int_{0}^{\phi_{s}} \frac{1}{E} \frac{D}{1-D} \mathrm{~d} x+\left.\frac{1}{k} \frac{d}{1-d}\right|_{\phi=\phi_{s}}=\left.\frac{1}{k_{1}} \frac{d_{1}}{1-d_{1}}\right|_{\phi=\phi_{s}}
$$

The expression of $H$ is given by equation (24) for $\phi_{s}<\phi^{*}$, and the expression of $h$ is obtained from the same equation for $\phi_{s}>\phi^{*}$. More details about the determination of $H$ and $h$ are given in Appendix D.

\section{Model description: $2 \mathrm{D} / 3 \mathrm{D}$ case}

In this section, the TLSV2 model is first extended to 2D/3D in subsection 4.1, then some details about the implementation are given in subsection 4.2 and 4.3. For simplicity all the equations are written for the $2 \mathrm{D}$ case, the extension to $3 \mathrm{D}$ being quite straightforward.

\section{1. $2 D / 3 D$ equations}

We consider a domain $\Omega$ with scalar isotropic bulk damage $D(\phi)$ and damage on the skeleton $d(\phi)$. The energy is:

$$
W(\boldsymbol{u}, \phi)=\int_{\Omega} \Psi(\boldsymbol{\epsilon}, D(\phi)) \mathrm{d} \Omega+\int_{\Gamma_{\mathrm{s}}(\phi)} \varphi\left(\llbracket \boldsymbol{u} \rrbracket, d\left(\phi_{s}\right)\right) \mathrm{d} \Gamma
$$

with

$$
\Psi(\boldsymbol{\epsilon}, D(\phi))=\frac{1}{2}(1-D) \boldsymbol{\epsilon}: \mathbb{C}: \boldsymbol{\epsilon}
$$

where $\mathbb{C}$ is the Hooke order 4 elasticity tensor, and

$$
\varphi\left(\llbracket \boldsymbol{u} \rrbracket, d\left(\phi_{s}\right)\right)=\frac{1}{2}\left(\frac{1}{d}-1\right) \llbracket \boldsymbol{u} \rrbracket \cdot \boldsymbol{k} \cdot \llbracket \boldsymbol{u} \rrbracket
$$

where $\boldsymbol{k}$ is the reference cohesive stiffness order 2 tensor.

In order to introduce an asymmetry between tension and compression, the potential (27) is modified [3]:

$$
\Psi(\boldsymbol{\epsilon}, D)=\mu\left(1-\alpha_{i} D\right) \epsilon_{i}^{2}+\frac{\lambda}{2}(1-\alpha D) \operatorname{tr}(\boldsymbol{\epsilon})^{2}
$$

where $\mu$ and $\lambda$ are the Lamé coefficients, $\epsilon_{i}$ the eigenvalues of the strain tensor and

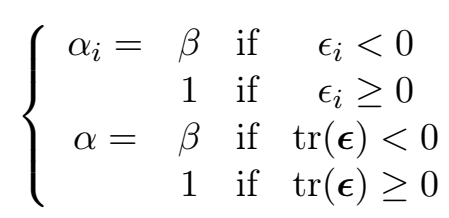




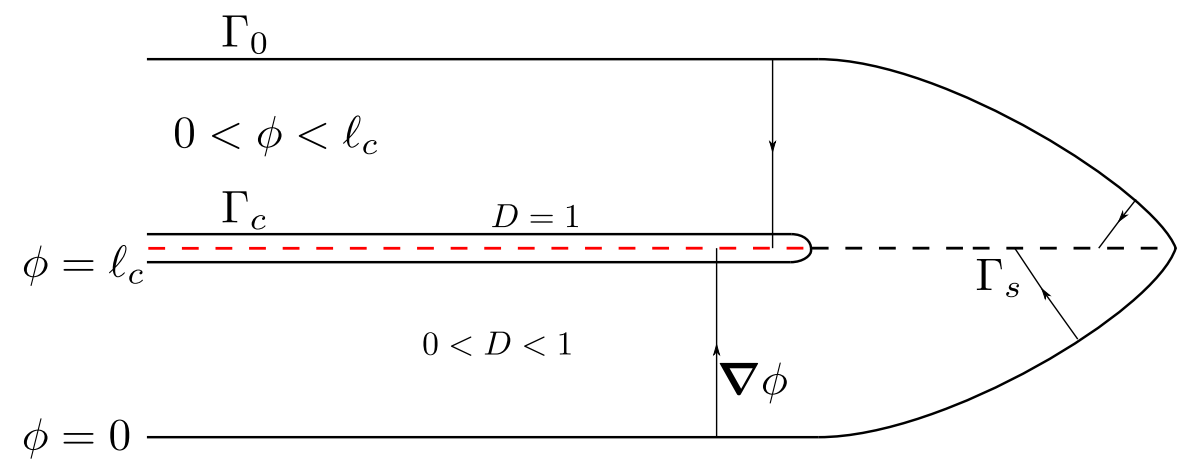

(a) TLSV1

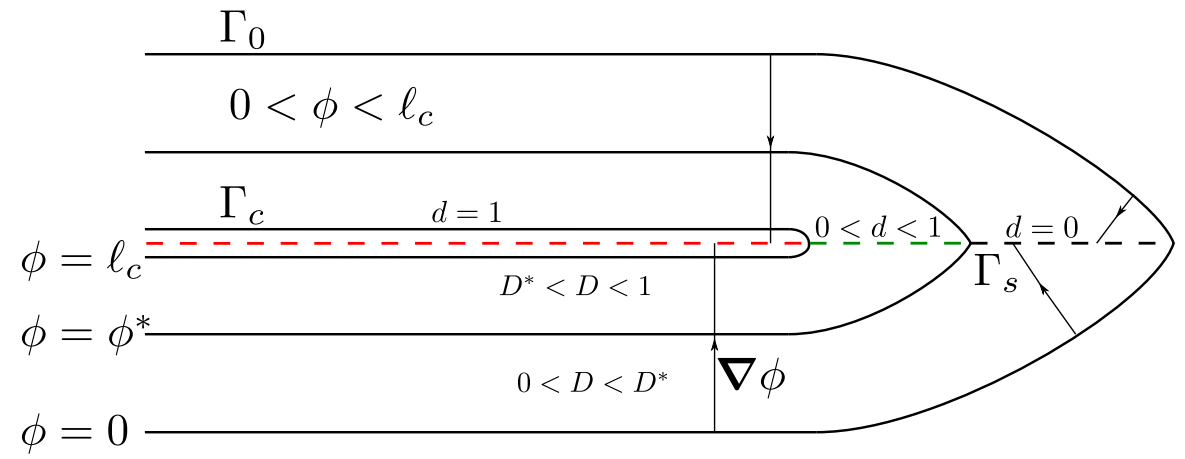

(b) TLSV2

Figure 10: Skeleton $\Gamma_{s}$ (dashed line) of the level-set in the TLS.

with $0 \leq \beta \leq 1$ a user defined parameter. State equations become:

$$
\left\{\begin{array}{l}
\sigma_{i}=2 \mu\left(1-\alpha_{i} D\right) \epsilon_{i}+\lambda(1-\alpha D) \operatorname{tr}(\boldsymbol{\epsilon}) \\
Y=-\mu \alpha_{i} \epsilon_{i}^{2}-\alpha \frac{\lambda}{2} \operatorname{tr}(\boldsymbol{\epsilon})^{2}
\end{array}\right.
$$

If $\beta=1$, the linear potential (27) is recovered. If $\beta=0$, the material recovers its stiffness in compression and damage can only grow in tension.

As explained in section 2.3, both bulk and interfacial models are governed by the unique variable $\phi$, which ranges from negative values (undamaged material), to values greater than $\ell_{\mathrm{c}}$ which corresponds to a traction free crack. It can be noticed that, contrary to classical damage approaches or the TLSV1 approach (cf. figure $10(\mathrm{a})$ ), there is no fully damaged zone since the bulk damage $D(\phi)$ never goes to one. Instead, a cohesive crack is expected to appear on the skeleton $\Gamma_{s}$ of the level set field $\phi$, which corresponds to the surface where $\boldsymbol{\nabla} \phi$ is discontinuous (cf. figure 10 (b)). Note that in the TLSV1, the position of the macro-cracks is determined by the zone where $\phi>\ell_{\mathrm{c}}$. The elements crossed the iso- $\ell_{\mathrm{c}}$ of the level-set field need some particular enrichment to introduce a discontinuity of the displacement field, since the width of the zone $\phi>\ell_{c}$ is not exactly zero, as explained by [3]. In the TLSV2, the position of the macro-cracks is given by the surface $\Gamma_{s}$, therefore the displacement jump can be modeled using a more classical Heaviside enrichment [27].

This paper will focus on symmetric problems across a line parallel to the $y$ axis, with loading conditions such that the position of the skeleton corresponds to the axis of symmetry (see figure 11). In that case, no enrichment is needed since the discontinuity of the displacement field is explicitly modeled. The non-symmetric case, with detection of the position and enrichment of the skeleton, will be studied in a forthcoming paper. A simple interfacial behavior will be considered, with no cohesive forces in the direction tangent to the crack faces; the reference cohesive stiffness $\boldsymbol{k}$ is assumed to have the following form: 


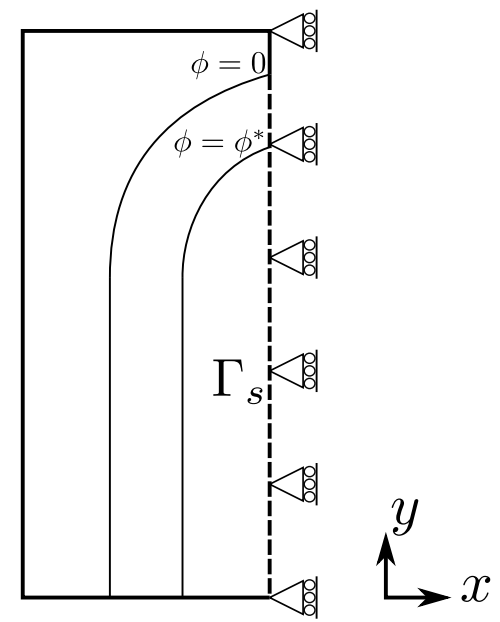

Figure 11: Location of the skeleton in the TLSV2, for a symmetric problem across a plane parallel to the $y$ axis.

$$
[\boldsymbol{k}]_{(\boldsymbol{x}, \boldsymbol{y})}=\left[\begin{array}{cc}
k_{n} & 0 \\
0 & 0
\end{array}\right]
$$

where $k_{n}$ is a positive constant.

Figure 12 depicts the cohesive forces on the deformed configuration. For $0<\phi_{s}<\phi^{*}, d=0$, the displacement jump is null. Then for $\phi^{*}<\phi_{s}<\ell_{\mathrm{c}}$, the displacement jump starts to increase, with cohesive forces on the crack lips. Finally, for $\phi_{s}>\ell_{c}$, there are no cohesive forces anymore. Note that in figures 10 and 12, the zone $\phi_{s}>\ell_{c}$ was represented with a certain thickness for clarity; however in practice the width of this zone is close to zero.

Within the TLS approach, the level set $\phi$ must fulfill the following condition [28]:

$$
\|\nabla \phi\|=1
$$

The time derivative of (34) gives:

$$
\nabla \dot{\phi} \cdot \nabla \phi=0
$$

meaning that $\dot{\phi}$ is constant along the gradient of $\phi$. Therefore, the driving fields of $\dot{\phi}$ must belong to the following space:

$$
\overline{\mathscr{Y}}=\left\{\bar{y} \in L^{2}\left(\Omega_{+}\right), \quad \nabla \bar{y} \cdot \nabla \phi=0\right\}
$$

where

$$
\Omega_{+}=\{\boldsymbol{x} \in \Omega, \phi(\boldsymbol{x})>0\}
$$

The space $\overline{\mathscr{Y}}$ is the space of the fields constant along the gradient of $\phi$ in the damaged zone $\Omega_{+}$. The computation of the configurational force $g$ and the critical value $g_{\mathrm{c}}$ has to be modified. These two quantities, which belong to $\overline{\mathscr{Y}}$, are now obtained as solutions of the following variational problems:

$$
\begin{gathered}
\int_{\Gamma_{0}} g Y^{*} \mathrm{~d} \Gamma=\int_{\Omega} Y D^{\prime} Y^{*} \mathrm{~d} \Omega+\int_{\Gamma_{s}} \frac{1}{2} y d^{\prime} Y^{*} \mathrm{~d} \Gamma, \forall Y^{*} \in \overline{\mathscr{Y}} \\
\int_{\Gamma_{0}} g_{\mathrm{c}} Y^{*} \mathrm{~d} \Gamma=\int_{\Omega} Y_{\mathrm{c}} H(D) D^{\prime} Y^{*} \mathrm{~d} \Omega+\int_{\Gamma_{s}} \frac{1}{2} y_{\mathrm{c}} h(d) d^{\prime} Y^{*} \mathrm{~d} \Gamma, \quad \forall Y^{*} \in \overline{\mathscr{Y}}
\end{gathered}
$$




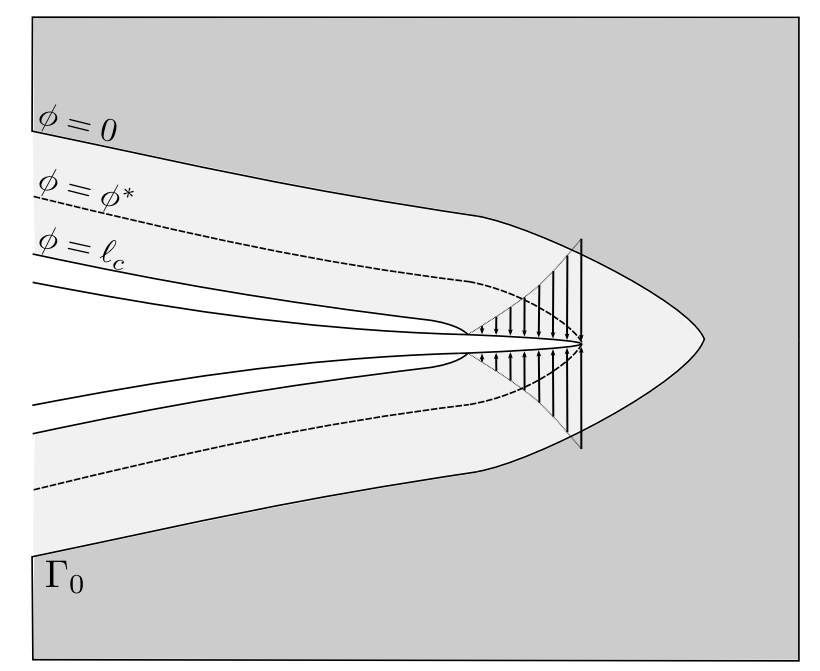

Figure 12: Illustration of the cohesive forces in the TLSV2, plotted on the deformed configuration.

These variational problems are solved using propagation modes as detailed in [30]. On top of the classical TLS terms we have additional terms on the skeleton. Note that the difference of $g$ on each side of the crack will only depend on the bulk damage part. It is the difference of strain bulk distribution which will decide the direction of the crack.

\subsection{Non-local fields computation}

The computation of $g$ and $g_{\mathrm{c}}$ from equation (38) and (39) involves bulk and interfacial contributions defined on the skeleton. In order to have a more generic formalism, we introduce non-local fields $\bar{X} \in \overline{\mathscr{Y}}$, computed from volumic contributions $X$ and lineic contributions $x$ defined on $\Gamma_{s}$ by solving the following equation:

$$
\int_{\Gamma_{0}} \bar{X} Y^{*} \mathrm{~d} \Gamma=\int_{\Omega} X D^{\prime} Y^{*} \mathrm{~d} \Omega+\int_{\Gamma_{s}} x d^{\prime} Y^{*} \mathrm{~d} \Gamma, \forall Y^{*} \in \overline{\mathscr{Y}}
$$

For instance, when computing $g, \bar{X}$ has to be replaced by $g, X$ by $Y$ and $x$ by $\frac{1}{2} y$ in equation (40).

The space of the non-local fields $\overline{\mathscr{Y}}$ is discretized using a basis of mode functions $\left(M_{i}\right)_{i \in M}(M$ being the set of the modes), whose construction is detailed in [30]. Any non-local field $\bar{X}$ can then be written in its discretized form as:

$$
\bar{X}^{h}(\boldsymbol{x})=\sum_{i \in M} \bar{X}_{i} M_{i}(\boldsymbol{x})
$$

Each coefficient $\bar{X}_{i}$ is then computed by injecting expression (41) into equation (40) and lumping the involved matrix:

$$
\bar{X}_{i}=\frac{\int_{\Omega} X D^{\prime} M_{i} \mathrm{~d} \Omega+\int_{\Gamma_{s}} x d^{\prime} M_{i} \mathrm{~d} \Gamma}{\int_{\Gamma_{0}} M_{i} \mathrm{~d} \Omega}, \forall i \in M
$$

In practice, equation (42) may be computed using a basic matrix-vector product. Indeed, the modes $M_{i}$ can be discretized over the basis $\left(L_{j}\right)_{j \in N}$ of the regular finite element interpolation functions:

$$
M_{i}(\boldsymbol{x})=\sum_{j \in N} M_{i j} L_{j}(\boldsymbol{x}), \quad \forall i \in M
$$

with $N$ the set of the finite element interpolation functions. The computation of the coefficients $\left(M_{i j}\right)_{\substack{i \in M \\ j \in N}}$ is explained in [30]. By noting $\{\overline{\boldsymbol{X}}\}=\left(\bar{X}_{i}\right)_{i \in M}$ and $[\boldsymbol{M}]=\left(M_{i j}\right)_{\substack{i \in M \\ j \in N}}$, coefficients $\bar{X}_{i}$ are computed with the following matrix-vector product: 


$$
\{\overline{\boldsymbol{X}}\}=([\boldsymbol{M}]\{\boldsymbol{A}\}) \cdot /([\boldsymbol{M}]\{\boldsymbol{B}\})
$$

where.$/$ is the element-wise division, and

$$
\begin{aligned}
A_{i} & =\int_{\Omega} X L_{i} D^{\prime} d \Omega+\int_{\Gamma_{s}} x L_{i} d^{\prime} \mathrm{d} \Gamma, \quad \forall i \in N \\
B_{i} & =\int_{\Omega} L_{i} d \Omega, \quad \forall i \in N
\end{aligned}
$$

\subsection{Equilibrium equations}

The weak form of the equilibrium of the structure consists of finding $\boldsymbol{u} \in \mathcal{C}$ such that:

$$
\int_{\Omega}(1-D) \boldsymbol{\epsilon}(\boldsymbol{u}): \mathbb{C}: \boldsymbol{\epsilon}\left(\boldsymbol{u}^{*}\right) \mathrm{d} \Omega+\int_{\Gamma_{s}} \boldsymbol{t}(\llbracket \boldsymbol{u} \rrbracket, d) \cdot \llbracket \boldsymbol{u}^{*} \rrbracket \mathrm{d} \Gamma=\int_{\Gamma_{d}} \boldsymbol{F}_{d} \cdot \boldsymbol{u}^{*} \mathrm{~d} \Gamma, \forall \boldsymbol{u}^{*} \in \mathcal{C}_{0}
$$

where $\boldsymbol{F}_{d}$ is the vector of the external loading, applied on the boundary $\Gamma_{d}$ and:

$$
\mathcal{C}=\left\{\boldsymbol{v} \in H^{1}\left(\Omega \backslash \Gamma_{s}\right), \boldsymbol{v}=\boldsymbol{u}_{d} \text { on } \Gamma_{u}\right\}
$$

with $\boldsymbol{u}_{d}$ a prescribed displacement on boundary $\Gamma_{u}$ and

$$
\mathcal{C}_{0}=\left\{\boldsymbol{v} \in H^{1}\left(\Omega \backslash \Gamma_{s}\right), \boldsymbol{v}=\mathbf{0} \text { on } \Gamma_{u}\right\}
$$

The cohesive part of the TLSV2 model, presented in section 2.1, has an infinite initial stiffness, which leads to ill-conditioned problems in the framework of a displacement-based formulation like (47). A first solution proposed by [4], called the Lagrangian formulation, allows to overcome this problem by considering two unknown fields, the displacement field $\boldsymbol{u}$ and the cohesive traction field $\boldsymbol{t}$ :

$$
\left\{\begin{array}{clll}
\int_{\Omega}(1-D) \boldsymbol{\epsilon}(\boldsymbol{u}): \mathbb{C}: \boldsymbol{\epsilon}\left(\boldsymbol{u}^{*}\right) \mathrm{d} \Omega+\int_{\Gamma_{s}} \boldsymbol{t} \cdot \llbracket \boldsymbol{u} \boldsymbol{u}^{*} \rrbracket \mathrm{d} \Gamma & =\int_{\Gamma_{d}} \boldsymbol{F}_{d} \cdot \boldsymbol{u}^{*} \mathrm{~d} \Gamma & , \forall & \boldsymbol{u}^{*} \in \mathcal{C}_{0} \\
\int_{\Gamma_{s}} \boldsymbol{t}^{*} \cdot\left(\llbracket \boldsymbol{u} \rrbracket-\frac{d}{1-d} \boldsymbol{k}^{-1} \cdot \boldsymbol{t}\right) \mathrm{d} \Gamma & =0 & , \forall & \boldsymbol{t}^{*} \in \mathcal{A}
\end{array}\right.
$$

where $\mathcal{A}$ is the space of the cohesive traction fields:

$$
\mathcal{A}=\left\{\boldsymbol{t}^{*} \in \mathbb{R}^{2}, \boldsymbol{t}^{*}=\left\{\begin{array}{c}
t_{n}^{*} \\
0
\end{array}\right\}, t_{n}^{*} \in \mathbb{R}\right\}
$$

Here, $\boldsymbol{k}^{-1}$ is not the classical inverse matrix of $\boldsymbol{k}$ (which is not invertible), but is defined as:

$$
\left[\boldsymbol{k}^{-1}\right]_{(\boldsymbol{x}, \boldsymbol{y})}=\left[\begin{array}{cc}
k_{n}^{-1} & 0 \\
0 & 0
\end{array}\right]
$$

The main drawback of this formulation is that it requires to evaluate the compliance operator $\frac{d}{1-d} \boldsymbol{k}^{-1}$, which tends to infinity when $d$ tends to 1 . In order to overcome this issue, a modified Lagrangian formulation is proposed in [4], by introducing an augmented cohesive traction $\boldsymbol{\lambda}$ :

$$
\boldsymbol{\lambda}=\boldsymbol{t}+\boldsymbol{k} \llbracket \boldsymbol{u} \rrbracket=\boldsymbol{k} \frac{\llbracket \boldsymbol{u} \rrbracket}{d}
$$

such that $y$ becomes:

$$
y=\frac{1}{2} \boldsymbol{\lambda} \cdot \boldsymbol{k}^{-1} \cdot \boldsymbol{\lambda}
$$

The weak form of the equilibrium equation is now:

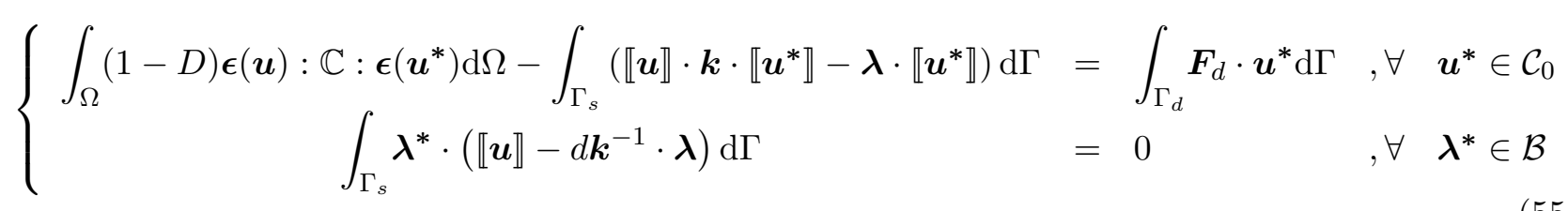


where $\mathcal{B}$ is the space of the augmented cohesive traction fields:

$$
\mathcal{B}=\left\{\boldsymbol{\lambda}^{*} \in \mathbb{R}^{2}, \boldsymbol{\lambda}^{*}=\boldsymbol{t}^{*}+\boldsymbol{k} \llbracket \boldsymbol{u}^{*} \rrbracket, \boldsymbol{t}^{*} \in \mathcal{A}, \boldsymbol{u}^{*} \in \mathcal{C}_{0}\right\}
$$

As the orientation of the $\boldsymbol{\lambda}$ field resulting from the resolution of (55) is arbitrary, the expression of $y$ given by equation (54) must be modified, in order to have the damage variable $d$ evolving only when the cohesive crack opens. Because of the particular form of $\boldsymbol{k}^{-1}$, the component $\lambda_{t}$ of $\boldsymbol{\lambda}$ along the $y$ direction is null, which gives:

$$
y=\frac{1}{2} k_{n}^{-1}\left\langle\lambda_{n}\right\rangle_{+}^{2}
$$

where $\lambda_{n}$ is the component of $\boldsymbol{\lambda}$ along the $x$ direction.

Since we do not need to evaluate any term tending to infinity, the formulation (55) is wellconditioned. Note that we can also use it to solve a purely cohesive problem (which corresponds to the case where the bulk damage variable $D$ is equal to zero).

\section{Numerical simulations}

In this section, after a brief explanation on how the model parameters were chosen (section 5.1), the different models presented so far are tested on numerical examples, in 1D (section 5.2) and in 2D (sections 5.3 and 5.4).

\subsection{Model parameters}

The 1D example is used to compare the numerical accuracy of the TLSV1 and the TLSV2. The TLSV2 model is chosen to be equivalent in 1D to the TLSV1 model, as explained in section 3.1. The local behavior of the material is mainly determined by the choice of the softening function $H$. Two different softening functions, represented on figure 14 with the corresponding local behavior, are considered:

- A constant function $H(D)=1$, which corresponds to the most abrupt case. In what follows we will refer to this model as the "abrupt model", referring to the form of the corresponding local behavior.

- A function $H$ which is computed in order to get a behavior in 1D of the TLSV1 equivalent to a CZM, according to [32] (the expression of $H$ is recalled in Appendix A). In what follows we will refer to this model as the "gradual model". The bilinear traction-separation law from [36], represented on figure 13, is used as a starting point.

For the 2D example, we consider only the gradual model, which is the more realistic, for the TLSV1 and the TLSV2 approaches, with the asymmetric potential defined by expression (29) and $\beta=0$. The comparison is also done with the pure CZM. The numerical results for the TLSV1 and TLSV2 are obtained by an explicit algorithm developed by [30] and briefly recalled in Appendix E. This algorithm was adapted to the CZM case in Appendix F.

Damage profiles $D_{1}, D$ and $d$ introduced in section 3.1 and plotted on figure 9, are used for the different simulations. The critical energy release rate $Y_{\mathrm{c}}$ is computed from equations (23) and (B.1).

\section{2. $1 D$ case}

We consider the case of a 1D bar in tension, already introduced in section 2 . Since the problem is symmetric, only half of the bar is modeled. The length of the bar is $L=0.1 \mathrm{~m}$, the characteristic length $\ell_{\mathrm{c}}=0.025 \mathrm{~m}$, and for the TLSV2 model, the critical value is $\phi^{*}=0.5 \ell_{\mathrm{c}}$. The material parameters are given in table 3 .

Figure 15 shows the local behaviors and cohesive laws corresponding to the abrupt and gradual models. For the cohesive law equivalent to the abrupt model, the traction-separation law has the following expression (as shown in [22]):

$$
\sigma=\sigma_{c}\left(1-\sqrt{\frac{w}{w_{c}}}\right)
$$




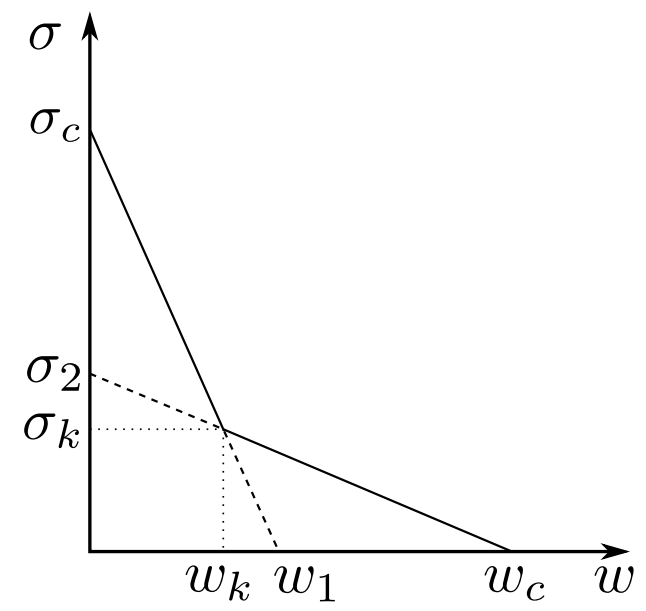

Figure 13: Cohesive bilinear law
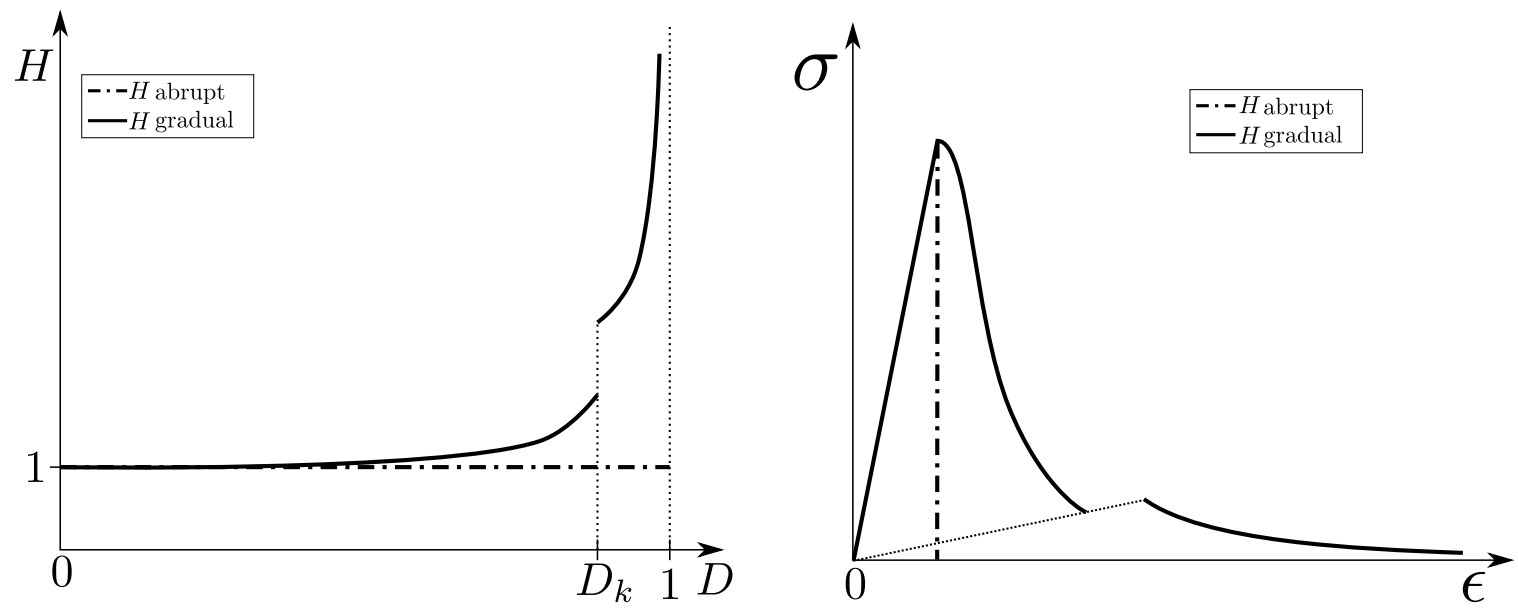

Figure 14: Hardening functions (left) and corresponding local behavior (right)

\begin{tabular}{|l|c|c|}
\hline Elastic parameters & $E(\mathrm{MPa})$ & 37200 \\
\hline \multirow{4}{*}{ Gradual model parameters } & $\sigma_{c}(\mathrm{MPa})$ & 3,5 \\
& $\sigma_{k}(\mathrm{MPa})$ & 0,8 \\
& $w_{c}(\mathrm{~mm})$ & 0,46 \\
& $w_{1}(\mathrm{~mm})$ & 0,0125 \\
\hline
\end{tabular}

Table 3: 1D tensile test: material parameters. 

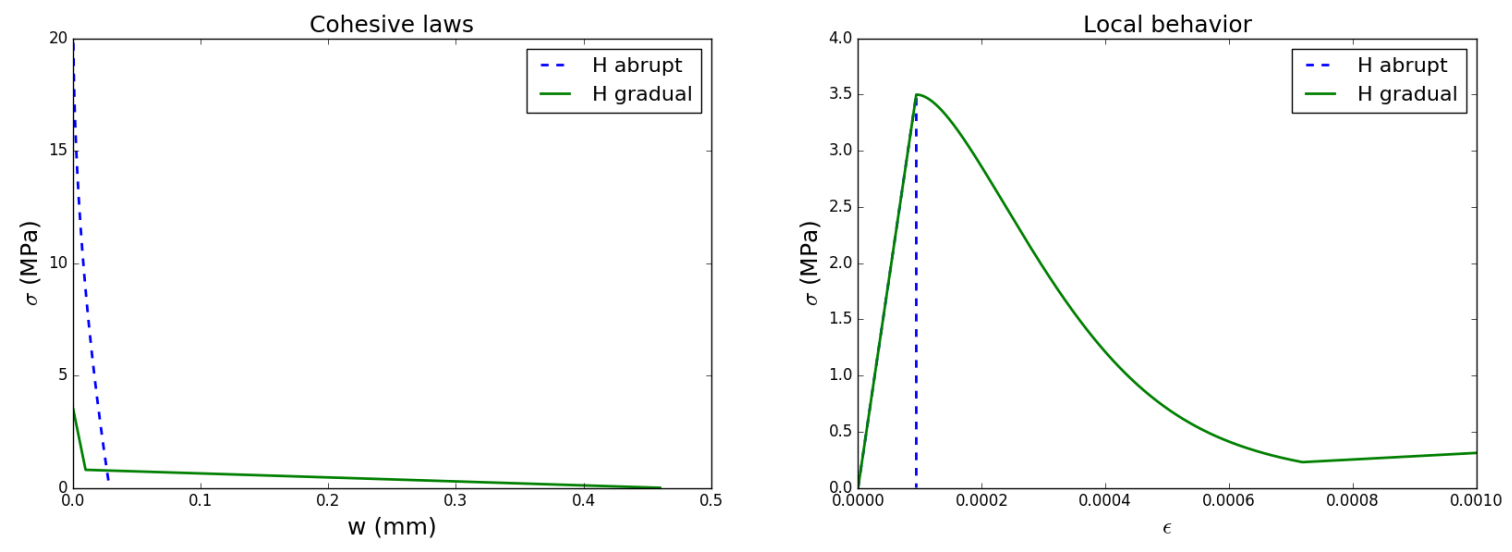

Figure 15: Cohesive laws (right) and corresponding local behavior (left)

The critical opening and stress $w_{c}$ and $\sigma_{c}$ have the following expressions:

$$
w_{c}=\frac{2 \sigma_{c} \ell_{c}}{E}
$$

and

$$
\sigma_{c}=\sqrt{\frac{3 E G_{F}}{2 \ell_{c}}}
$$

with $G_{F}$ the fracture energy, corresponding to the area under the curve $\sigma=f(w)$. The tractionseparation for the abrupt model plotted on figure 15 was determined for the same value of $\ell_{\mathrm{c}}$ used for the gradual model, $\sigma_{c}$ and $w_{c}$ being computed in order to have the same area under both curves (same energy). One can see that in order to keep the same fracture energy, the tensile strength of the abrupt model must be higher.

The stress versus mean strain $\left(\bar{\epsilon}=\frac{u(x=L)}{L}\right)$ curves for the solutions obtained numerically with the TLSV1 and TLSV2 approaches are plotted on figure 16, for both the abrupt and gradual models. The TLSV1 was tested for element sizes $h_{e}$ ranging from $\frac{\ell_{\mathrm{c}}}{5}$ to $\frac{\ell_{\mathrm{c}}}{300}$, and the TLSV2 with an element size of $\frac{\ell_{c}}{5}$. Results are compared with the TLSV1 analytical solution. For simplicity, it is denoted "Exact TLS solution" in the different figures, without specifying that it is for the version 1, since in 1D the two versions of the TLS are supposed to be equivalent in terms of global behavior.

It can be observed that in all the cases, the TLSV2 allows to recover the analytical solution for a relatively coarse mesh element size. On the contrary, for the abrupt model and coarse refinement, the TLSV1 captures the analytical solution at the beginning of the computation (see figure 16 (a)), but deviates from it at the end (see figure 16 (c)), that is to say when $D \rightarrow 1$. When the mesh is refined, the TLSV1 seems to converge non-uniformly toward the analytical solution, the convergence being fast at the beginning of the stress-strain curve, and slow at the end. A similar problem can be observed for the gradual model (see figure 16 (b) and (d)). As the softening function $H$ has been calculated to have a 1D global behavior of the TLS equivalent to a bilinear CZM, the analytical solution is piecewise linear, with a first elastic branch, followed by two decreasing branches which correspond to the traction-separation law depicted on figure 13. When the mesh is too coarse (figure 16 (b) and (d) for $h_{e}=\ell_{\mathrm{c}} / 5$, blue curve), the TLSV1 can not represent the change of slope of the analytical solution. This can be explained by the form of the hardening function $H$ of the gradual model (see figure 14), which is discontinuous for a certain value $D_{k}$ of the damage variable. This discontinuity stems from the discontinuity of the derivative at $w=w_{k}$ of the traction-separation law. In the TLS approach, it is necessary at some point to compute $\bar{H}$ (equation (8)), which requires to compute numerically an integral where $H$ appears, using a classical Gauss quadrature. With the parameters from table 3, $\phi_{k}=0.91$ (where $\phi_{k}=D^{-1}\left(D_{k}\right)$ ), which means that the zone $\left[\phi_{k}, \phi_{s}\right]$ has a width around $\ell_{c} / 10$. If the mesh used is too coarse (for instance for an element size $h_{e}=\ell_{\mathrm{c}} / 5$ in figure 16 (b) and (d)), there may be no Gauss point in the zone $\left[\phi_{k}, \phi_{s}\right]$, leading to an underestimated value of $\bar{H}$. For an element 


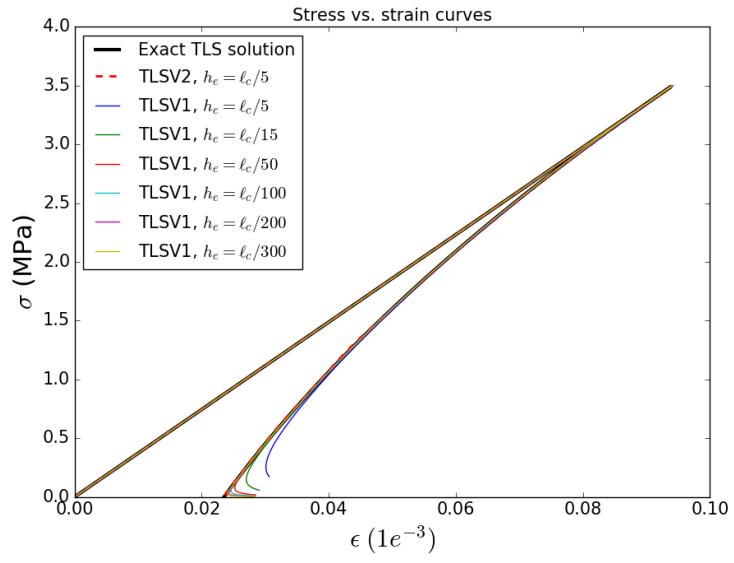

(a)

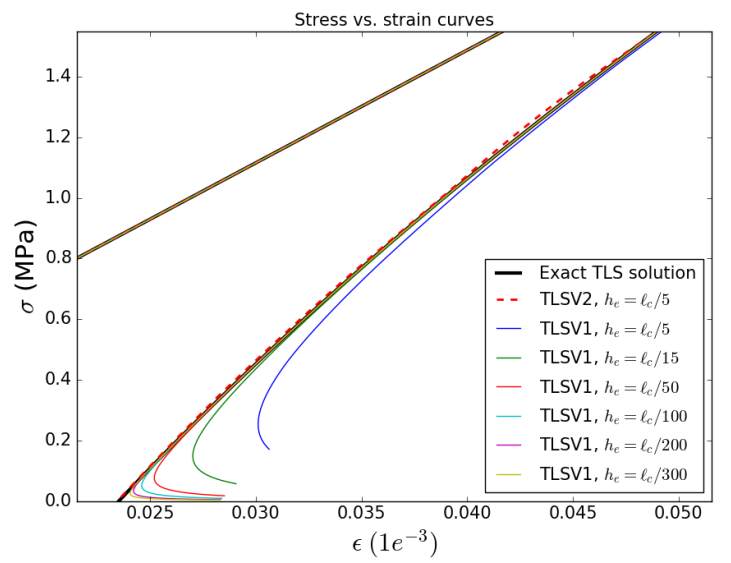

(c)

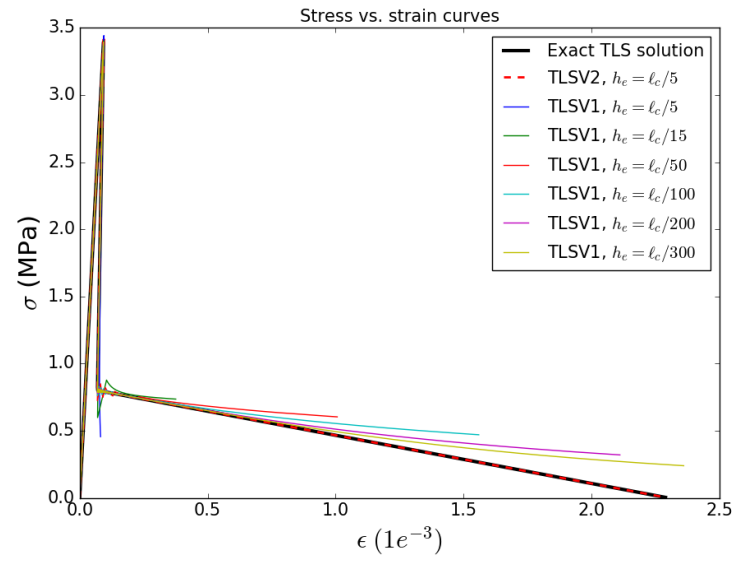

(b)

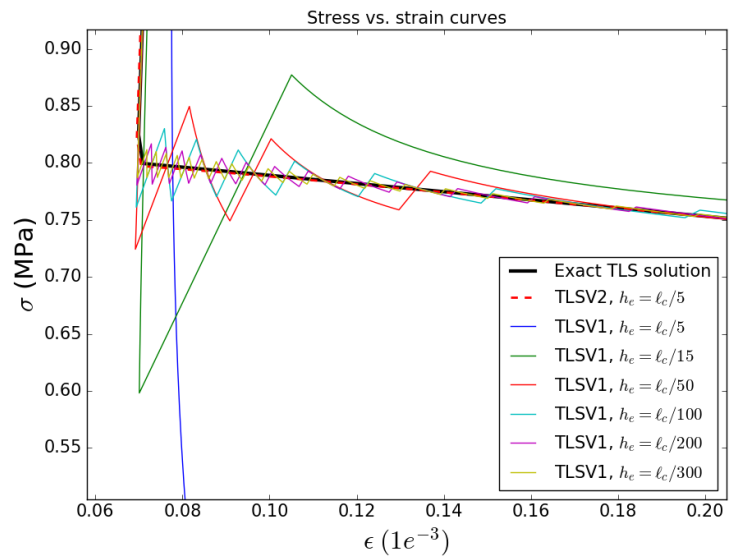

(d)

Figure 16: 1D tensile test: stress vs mean strain curves. Black thick lines correspond to the analytical solutions, other curves are obtained numerically. (a) Abrupt model. (b) Gradual model. (c) Abrupt model, zoom on the end of the computation. (d) Gradual model, zoom around the kink point of the curve. With both the abrupt and gradual model, the analytical and numerical TLV2 solutions are superimposed, for a relatively coarse mesh element size.

size greater than $h_{e}=\ell_{\mathrm{c}} / 15$, the mesh is fine enough to have at least one Gauss point in the zone $\left[\phi_{k}, \phi_{s}\right]$, therefore a change of slope of the solution in the numerical solution can be observed, even if the numerical solution is still far from the analytical one. With the TLSV2, since $\phi^{*}=0.5<\phi_{k}$, the tedious part of the integration of $H$ is taken into account by the cohesive contribution of the model, therefore the numerical results are close to the analytical solution even with a relatively coarse mesh.

The results that have been presented so far show that the convergence of the TLSV1 is particularly slow compared to the TLSV2. Whether it is for the abrupt or the gradual model, the finer the mesh and the further is the point where the numerical solution deviates from the analytical one. However, even for $h_{e}=\ell_{\mathrm{c}} / 300$, the last point of the numerical curve is still far from the analytical one. Some oscillations can also be noticed with the gradual model (see figure $16(\mathrm{~d})$ ), which correspond to finite elements passing into the zone $\left[\phi_{k}, \phi_{s}\right]$.

The results presented on this 1D example reveal a non-uniform convergence of the TLSV1, the convergence speed being very slow when the damage variable $D$ tends to 1 . This phenomenon stems from numerical difficulties which arise when computing the averaged quantities $\bar{Y}$ and $\bar{H}$ in (8):

- The computation of $\bar{Y}$ involves the local quantity $Y$, which is proportional to the square of the deformation $\epsilon$, which tends to infinity when the bulk damage variable $D$ tends to 1 .

- The used softening function $H$ may have a tedious behavior when $D$ tends to 1 , for instance it may tend to infinity. This is the case with the gradual model used in this section.

These problems come when $D \rightarrow 1$, and this is one of the reasons why the TLSV2 was introduced: 

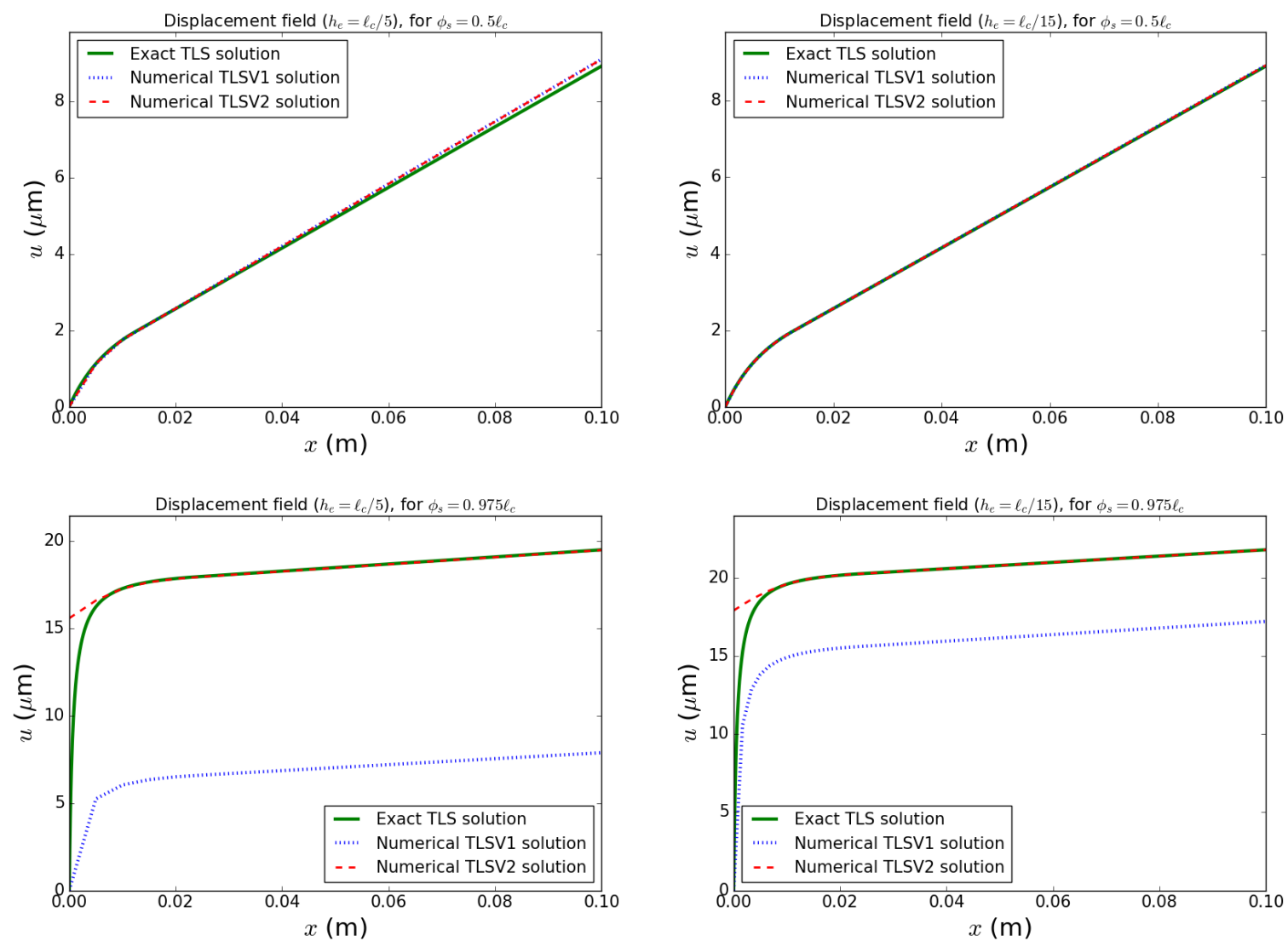

Figure 17: 1D tensile test: displacement solutions for the gradual model, at two loading stages (from top to bottom), for a finite element size of $h_{e}=\ell_{\mathrm{c}} / 5$ (left) and $h_{e}=\ell_{\mathrm{c}} / 15$ (right).

indeed, by restricting the bulk damage variable to values strictly smaller than 1 (see equation (13) and figure 6), the first terms of $g$ and $g_{\mathrm{c}}$ in equations (14) and (15), which come from the TLSV1, can now be integrated numerically with classical integration schemes, while the second terms, which come from the cohesive part of the model, are finite quantities which are directly evaluated on the cohesive zone.

Displacement solutions at two loading stages for the gradual model (conclusions are similar for the abrupt model), are plotted on figure 17. Around $x=0$, the TLSV2 is of course different from the TLSV1 analytical solution, since the kinematics of the two models differ. However, far enough from $x=0$, the TLSV2 is almost superimposed with the analytical solution, contrary to the TLSV1 which is quite far. The differences between the TLSV1 and the analytical solution increase as $\phi_{s}$ tends to $\ell_{\mathrm{c}}$.

The displacement error for $\phi_{s}=0.9999 \ell_{\mathrm{c}}$, defined as the relative error on the displacement $u(x=L)$ between the TLSV1/V2 and the analytical solution, is plotted as a function of the mesh element size for both the abrupt and gradual model on figure 18. For both models, the TLSV2 exhibits much lower error levels than the TLSV1. With the abrupt model (figure 18 (a)), the TLSV2 seems to have a better convergence rate, the error curve being steeper than the one of the TLSV1. However, with the gradual model (figure 18 (b)), the difference of slope between the error curves of TLSV1 and TLSV2 is not so obvious. In order to understand this phenomenon, a few details about the implementation of the TLS approach need to be recalled (more details can be found in Appendix E).

At each computation step $n$, the $1 \mathrm{D}$ problem is solved for a reference loading $F_{\text {ref }}$ (which can be either an imposed displacement or loading), giving a reference displacement solution $u_{\text {ref }}^{n}$. Then the actual displacement solution is obtained by multiplying this reference solution by a load factor, computed as:

$$
\mu^{n}=\sqrt{\frac{b^{n}}{a^{n}}}
$$


with

$$
\left\{\begin{array}{l}
a^{n}=\bar{Y}_{\mathrm{ref}}^{n} \\
b^{n}=Y_{\mathrm{c}} \bar{H}_{1}^{n}
\end{array}\right.
$$

for the TLSV1 and

$$
\left\{\begin{array}{l}
a^{n}=g_{\mathrm{ref}}^{n} \\
b^{n}=g_{c}^{n}
\end{array}\right.
$$

for the TLSV2.

Equation (61) shows that the load factor (and therefore, the displacement at the end of the bar) is function of a ratio between:

- A configurational force at the denominator, which is computed by integrating a quantity depending on the strain $\epsilon$

- A critical value numerator, which requires to compute the integral for the softening function $H_{1}$ (for the TLSV1) or $H$ (for the TLSV2)

It has been explained previously that these quantities are difficult to integrate with the TLSV1, since they both involve quantities which tend to infinity when $D$ tends to 1 , and how the TLSV2 deals with this problem. For the abrupt model, with the TLSV1, since the hardening function $H_{1}$ is constant, the numerator $b^{n}$ in equation (61) is exactly computed, so the only source of error is the computation of $a^{n}$. The displacement field being approximated with linear finite element, the quality of the approximation of the strain field which tends to infinity when $D$ tends to 1 is very poor. The computation of $b^{n}$ is optimal and the computation of $a^{n}$ does not converge, therefore the convergence rate of the TLSV1 is close to zero. On the contrary, with the TLSV2, $a^{n}$ is properly computed, so a good convergence rate is recovered.

With the gradual model, the numerator $b^{n}$ also has to be computed numerically. However, although it also involves quantities tending to infinity, these quantities are not approximated (contrary to the strain field). This means that when the mesh is refined, the error on $b^{n}$ decreases, which is not the case for $a^{n}$ as it was shown with the abrupt model. Here the computation of $a^{n}$ does not converge, but the computation of $b^{n}$ slightly increases when the mesh is refined, this is why in figure 18 (b), the error for the TLSV1 seems to decrease a bit more than in figure 18 (a).

Because of the dependence of the load factor to the ratio of two numerically difficult to integrate quantities, it seems difficult to draw some conclusions on the convergence rate of the TLS approach. However, it is evident that the TLSV2 allows to greatly decreases the error levels compared to the TLSV1.

\subsection{Three-points bending test}

As explained in section 4, this paper will focus on symmetric examples. The notched beam from [12] and the unnotched beam from [18] are considered. The dimensions of the notched beam are: length $L=500 \mathrm{~mm}$, distance between support $D=400 \mathrm{~mm}$, height $c=100 \mathrm{~mm}$, thickness $e=50 \mathrm{~mm}$, notch depth $t=20 \mathrm{~mm}$. For the unnotched beam, $L=1088 \mathrm{~mm}, D=L, c=500 \mathrm{~mm}$ and $e=40 \mathrm{~mm}$. The characteristic lengths come from [12] and [33]; $\ell_{\mathrm{c}}=8 \mathrm{~mm}$ for the notched beam was used by [12] with a non-local integral Mazars damage model, and $\ell_{\mathrm{c}}=20 \mathrm{~mm}$ for the unnotched beam was used by [33] with the TLSV1 to fit the experimental results from [18]. For the notched beam, the width of the notch is $\ell_{\text {notch }}=1 \mathrm{~mm}$. The simulations are performed under plane strain assumption. The material parameters used for the two tests are given in table 4 . The fracture energy $G_{F}$, computed as the area under the curve $t=f(w)$ is:

$$
G_{F}=\frac{1}{2}\left(\sigma_{c} w_{k}+\sigma_{k} w_{c}\right)=\frac{1}{2}\left(\sigma_{c} w_{1}+\sigma_{k}\left(w_{c}-w_{1}\right)\right)
$$

It is recalled that this paper focuses only on symmetric cases, where the position of the skeleton corresponds to the axis of symmetry (see section 4.1). Since the considered examples are symmetric, only half of the domain is modeled for each of them, using symmetry boundary conditions (see figure 


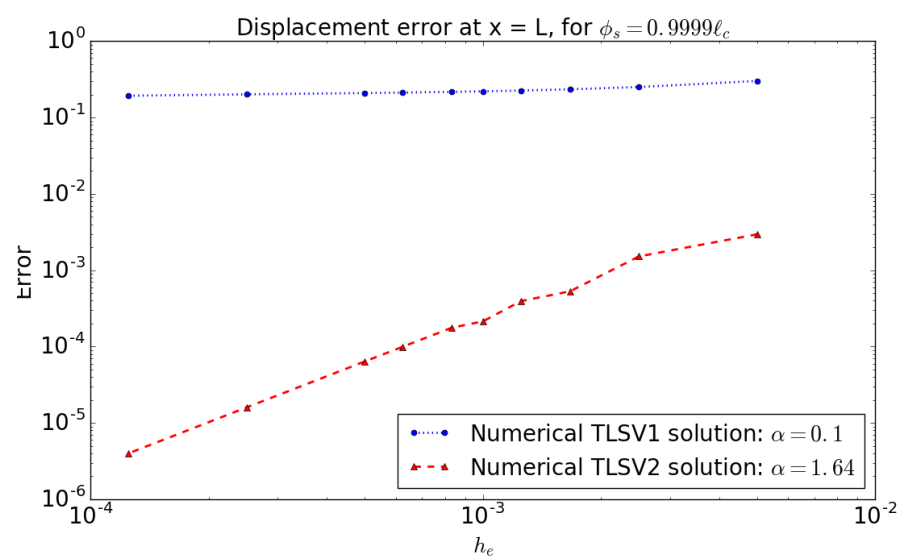

(a)

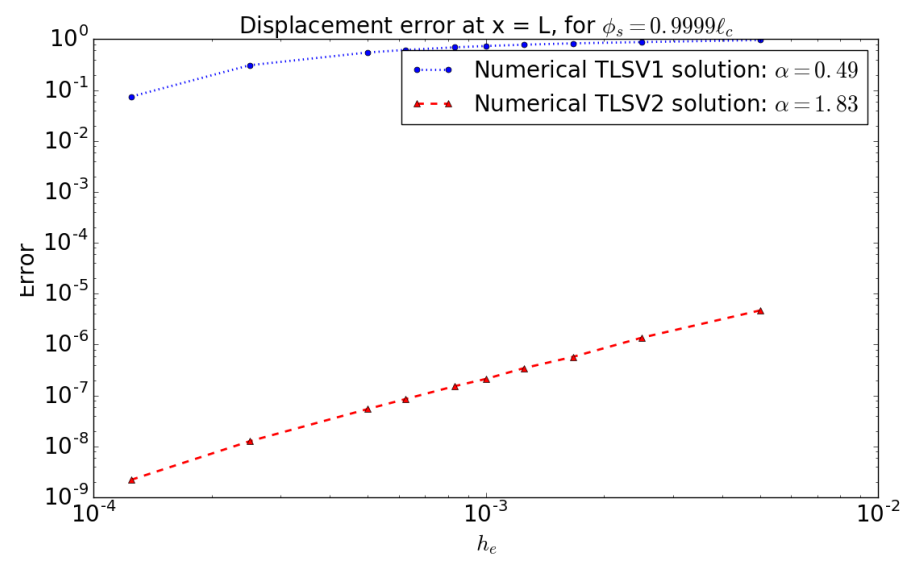

(b)

Figure 18: 1D tensile test: displacement convergence for the abrupt model (a) and for the gradual model (b) ( $\alpha$ : slope of the convergence curves).

\begin{tabular}{|l|c|cc|}
\cline { 3 - 4 } \multicolumn{2}{c|}{} & Notched beam & Unnotched beam \\
\hline \multirow{4}{*}{ Elastic parameters } & $E(\mathrm{MPa})$ & 30000 & 37000 \\
& $\nu$ & 0.24 & 0.21 \\
\hline \multirow{5}{*}{ Gradual model parameters } & $\sigma_{c}(\mathrm{MPa})$ & 4.0 & 4.2 \\
& $\sigma_{k}(\mathrm{MPa})$ & 0,26 & 1,5 \\
& $w_{c}(\mathrm{~mm})$ & 0,03 & 0,04 \\
& $w_{1}(\mathrm{~mm})$ & 0,021 & 0,0235 \\
& $G_{F}\left(\mathrm{~N} \cdot \mathrm{m}^{-1}\right)$ & 43,2 & 61,8 \\
\hline
\end{tabular}

Table 4: Three-points bending tests: material parameters. 


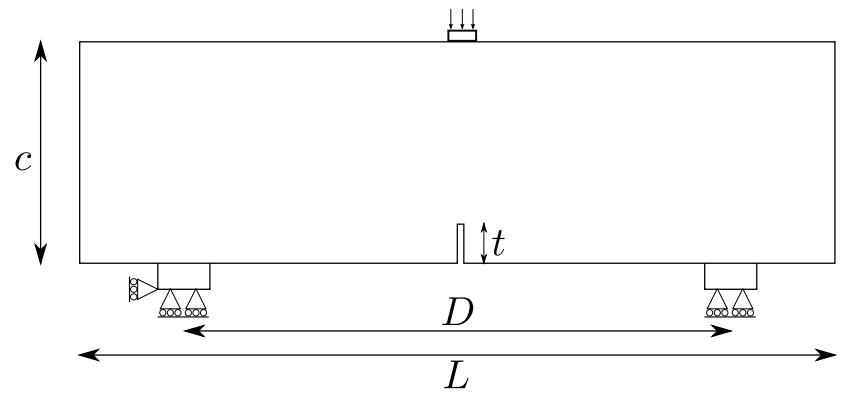

Figure 19: Three-points bending test on notched beam: geometry.

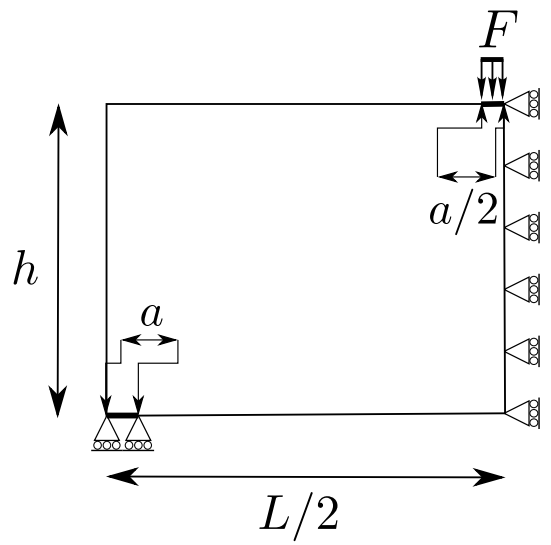

TLSV1

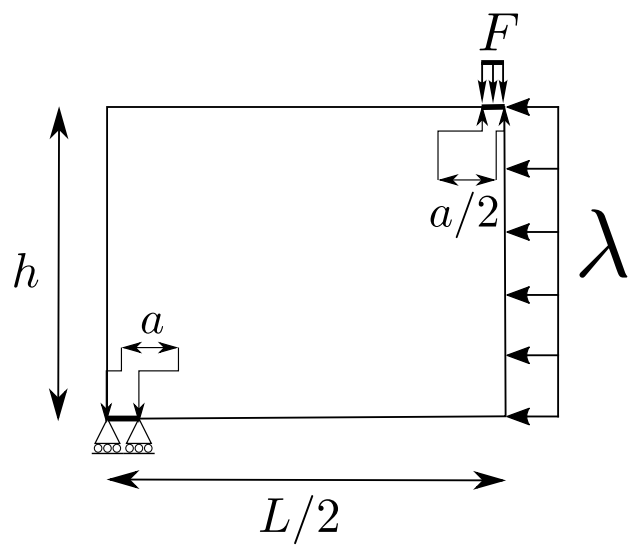

CZM, TLSV2

Figure 20: Three-points bending test on notched beam: boundary conditions.

20). The loading is applied on a line on the upper boundary of the beam, and the vertical displacement of a line on the lower boundary at the corner is locked. These two lines have the same width, which is of $a=5 \mathrm{~mm}$ for the notched beam and $a=60 \mathrm{~mm}$ for the unnotched beam. Note that in the case of the TLSV1, the displacement of the axis of symmetry is locked by fixing its displacement along the $x$ direction to zero (except for the parts were $\phi \geq \ell_{c}$ ), whereas for the TLSV2 and the CZM, the displacement along $x$ of the axis of symmetry is handled via the modified Lagrangian formulation presented in section 4.3 .

The bulk and cohesive damage fields as well as the cohesive forces are plotted on figure 21 . When $\phi<0$, both $D$ and $d$ are null. Then, for $0<\phi<\phi^{*}, D$ starts to grow whereas $d$ is still equal to zero. Finally for $\phi>\phi^{*}, d$ starts to grow, while the traction forces start to decrease, until $\phi \geq \ell_{\mathrm{c}}$.

Figure 22 shows for both tests the force-CMOD curves obtained with the cohesive, the TLSV1 and the TLSV2 models, for a first mesh where the element size is $h_{e}=\ell_{\mathrm{c}} / 5$. Note that the CMOD is defined as twice (because of the symmetric boundary conditions) the horizontal displacement of a point located at coordinates $\left(-\ell_{\text {notch }}, 0\right)$ for the notched beam, and $(-81,0)$ for the unnotched beam (units are $\mathrm{mm}$ ), assuming that the coordinates of the point located at the middle of the beam on the bottom boundary are $(0,0)$.

The TLSV1 dissipates more energy than the other two models, and shows oscillations with a high amplitude. On the contrary, the TLSV2 oscillates much less, and gives results which are very similar to the CZM. This last point is quite interesting: indeed, the parameters of the three models were computed in section 3 in order to have equivalent global behaviors in $1 \mathrm{D}$, but there is a priori no reasons that it is still the case in $2 \mathrm{D}$. It was shown numerically in [32] that for $\ell_{\mathrm{c}} \rightarrow 0$, the results of the TLSV1 tends to the results obtained by the CZM, but this requires small values of $\ell_{\mathrm{c}}$ and therefore, 

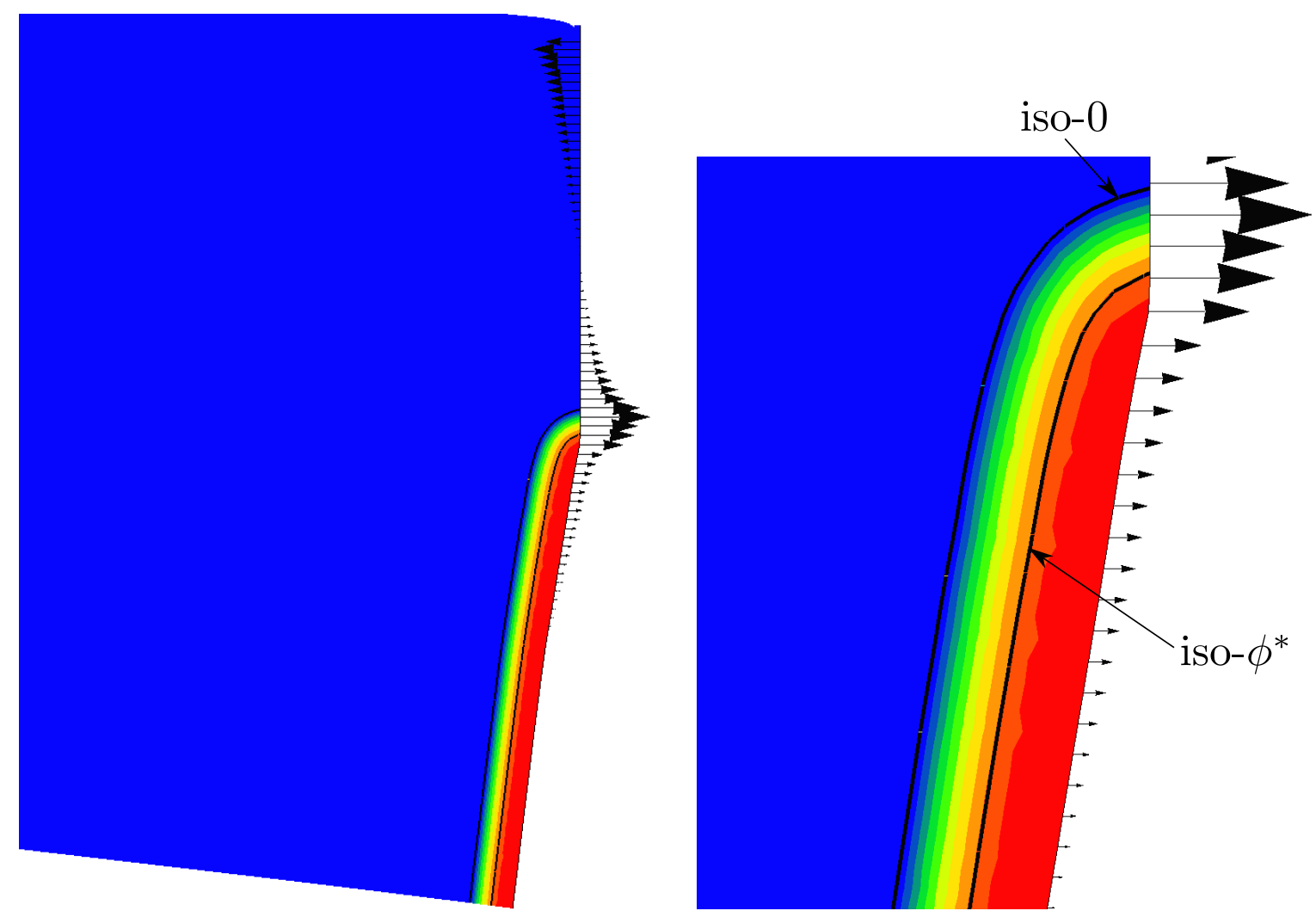

(a)

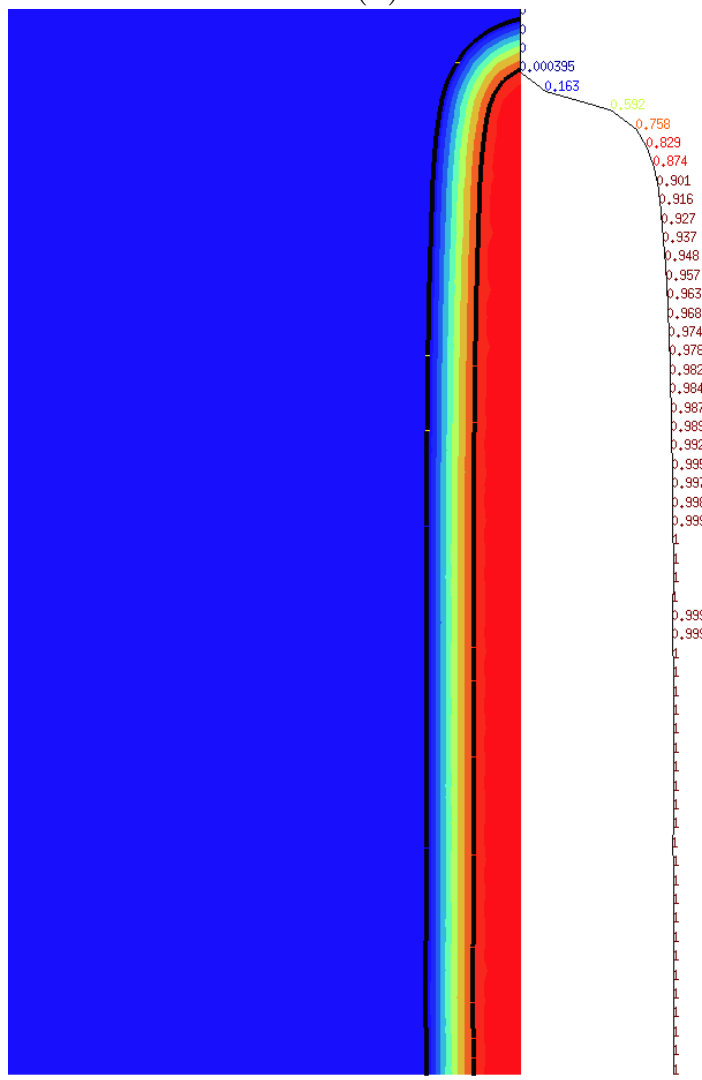

(b)

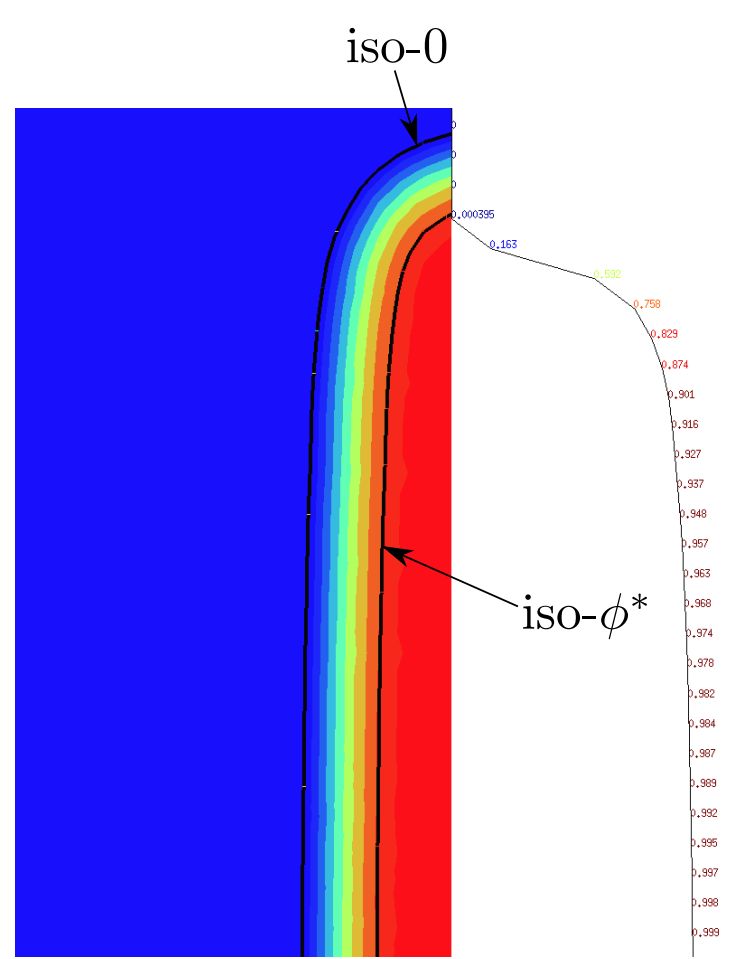

(c)

(d)

Damage

0,458 0.917

Figure 21: Three-points bending tests. The color map corresponds to the bulk damage field. (a) deformed configuration, traction forces (arrows) (b) deformed configuration, zoom (c) initial configuration, cohesive damage (line with numeric cohesive damage values on the right) (d) initial configuration, zoom. The black thick lines are the iso-zero and iso- $\phi^{*}$ of the level set field. 

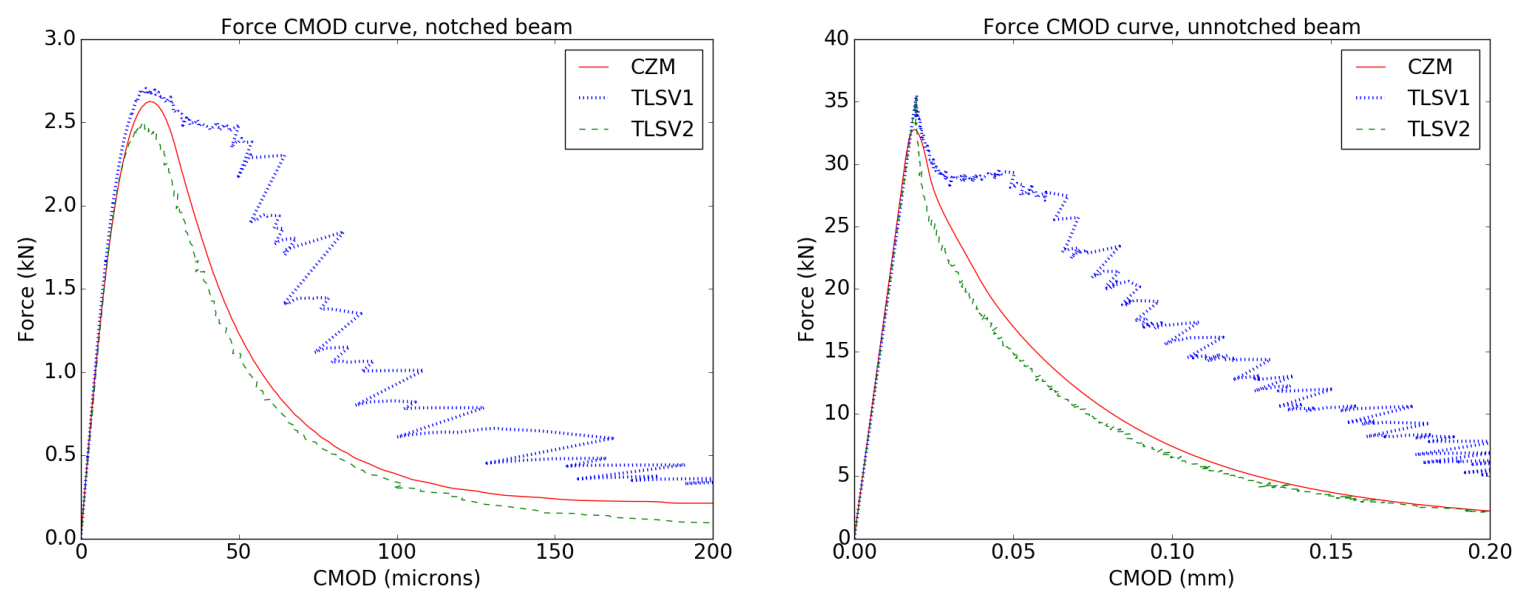

Figure 22: Three-points bending tests on notched (left) and unnotched (right) beam: force CMOD curves, comparison between the cohesive, the TLSV1 and the TLSV2 models, for a mesh where the element size is $h_{e}=\ell_{\mathrm{c}} / 5$.

very fine meshes.

Figure 23 shows for both tests the force CMOD curves obtained with the TLSV1, for element sizes of $\ell_{\mathrm{c}} / 5, \ell_{\mathrm{c}} / 10$ and $\ell_{\mathrm{c}} / 20$. The amplitude of the oscillations decreases as the mesh is refined, while the results seem to converge. However, this convergence is slow. It could be expected that the results of the TLSV1 should converge toward the results of the TLSV2, however as shown in the 1D example of section 5.2, it would require a huge number of elements in 2D. Note that in previous papers about the TLS approach, apparently better results have been obtained with the TLSV1. However, it must be emphasized that these results were obtained:

- For a constant softening function $H=1[28,3,40]$, which removes the difficulty of computing numerically $\bar{H}$.

- For very fine meshes, with an element size of at least $\ell_{\mathrm{c}} / 20$, as in [32].

On the contrary, the results obtained with the TLSV2 (figure 24) almost do not depend on the size of the elements of the mesh. Some oscillations can be observed at the beginning of the computation as the mesh is refined in the case of the notched beam. This is due to the explicit resolution algorithm, which strongly depends on the mechanical fields at a local scale. Refining the mesh in the case of the unnotched beam, which presents a stress singularity, tends to accentuate this effect, causing some oscillations.

Table 5 summarizes the dissipated energy for the different computations for the notched beam example. The reference value is computed as:

$$
W_{d}=e(c-t) G_{F}
$$

The numerical values are computed as the area under the applied load versus vertical displacement curves. Note that this analysis could not be performed for the unnotched beam example, because of the used boundary conditions. Indeed, for this example, a steel plate was used to apply the loading, resulting in branching of the crack when it reaches the top of the beam. Therefore, the dissipated energy which would be numerically computed would not correspond to the one which would be computed analytically with equation (67) (which corresponds to the case where the crack cuts the beam in half, straight through its section).

As expected, with the TLSV1 the dissipated energy is quite far from the reference value, and converges very slowly. It is much closer with the TLSV2, even if it seems a bit smaller than the value obtained with the CZM. As explained in section 5.1, the parameters of the TLSV1, TLSV2 and CZM models were chosen in order to have equivalent behaviors, and in particular the same dissipation. This is analytically true only in $1 \mathrm{D}$, and has no reason to be true in $2 \mathrm{D}$ or $3 \mathrm{D}$. A possible explanation is 

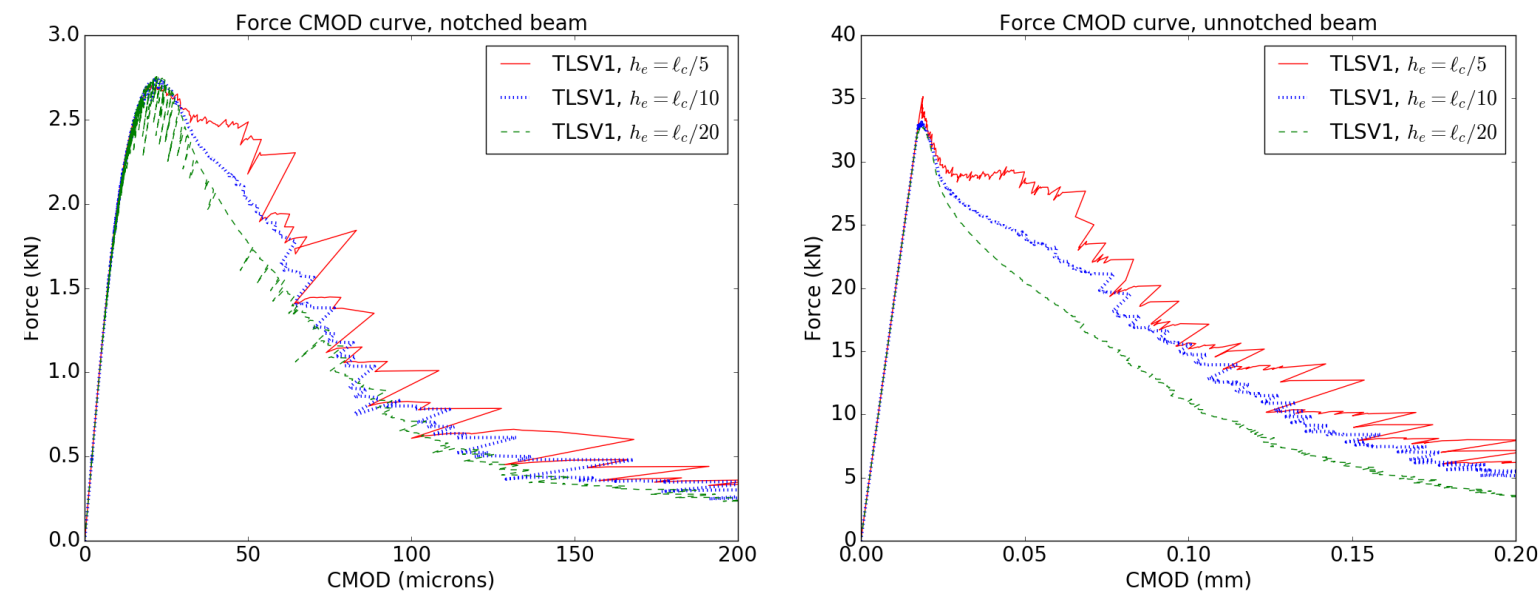

Figure 23: Three-points bending tests on notched (left) and unnotched (right) beam: force CMOD curves, obtained with the TLSV1 method, for several meshes.
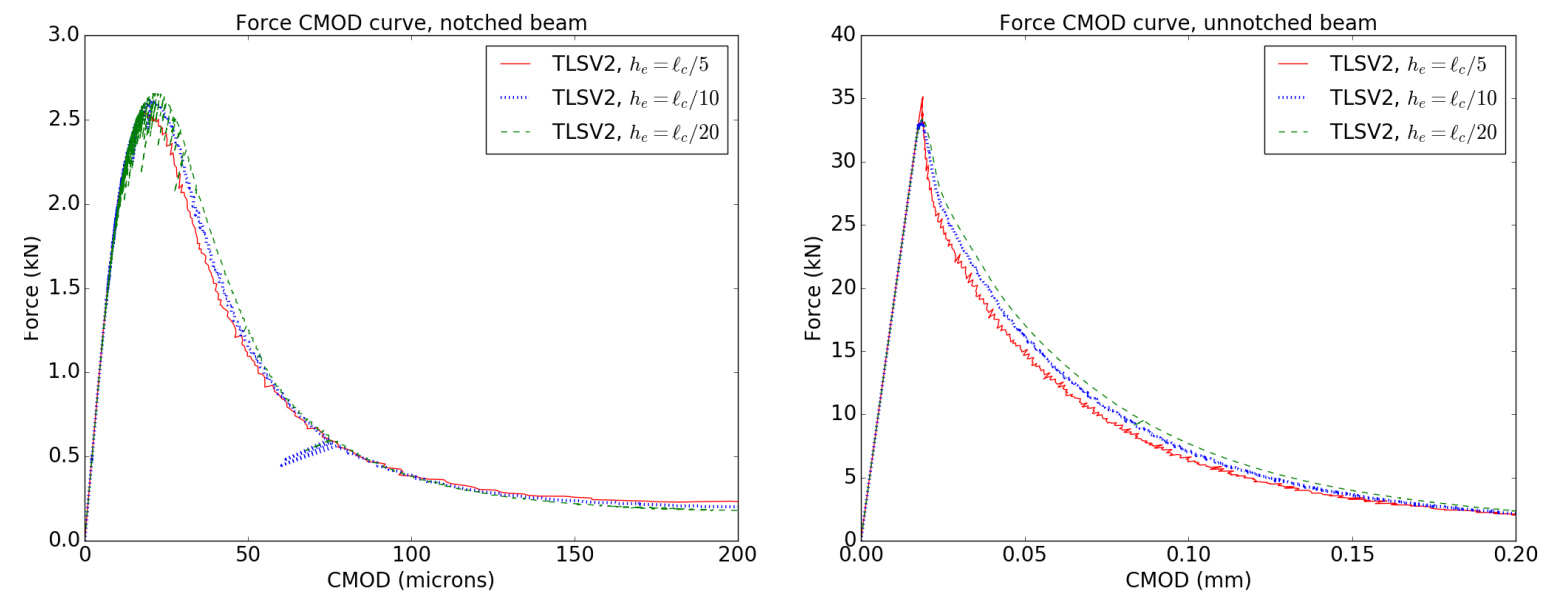

Figure 24: Three-points bending tests on notched (left) and unnotched (right) beam: force CMOD curves, obtained with the TLSV2 method, for several meshes.

\begin{tabular}{|l|c|c|}
\cline { 2 - 3 } \multicolumn{1}{c|}{} & Mesh element size & Dissipated energy \\
\hline \multirow{3}{*}{ CZM } & $h_{e}=\ell_{\mathrm{c}} / 5$ & 156,76 \\
& $h_{e}=\ell_{\mathrm{c}} / 10$ & 162,95 \\
& $h_{e}=\ell_{\mathrm{c}} / 20$ & 170,95 \\
\hline \multirow{3}{*}{ TLSV1 } & $h_{e}=\ell_{\mathrm{c}} / 5$ & 275,32 \\
& $h_{e}=\ell_{\mathrm{c}} / 10$ & 239,14 \\
& $h_{e}=\ell_{\mathrm{c}} / 20$ & 219,88 \\
\hline \multirow{3}{*}{ TLSV2 } & $h_{e}=\ell_{\mathrm{c}} / 5$ & 154,99 \\
& $h_{e}=\ell_{\mathrm{c}} / 10$ & 157,08 \\
& $h_{e}=\ell_{\mathrm{c}} / 20$ & 163,99 \\
\hline \multicolumn{2}{|c|}{ Reference value } & 172,8 \\
\hline
\end{tabular}

Table 5: Three-points bending tests (notched beam): dissipated energy (units are N.mm). 


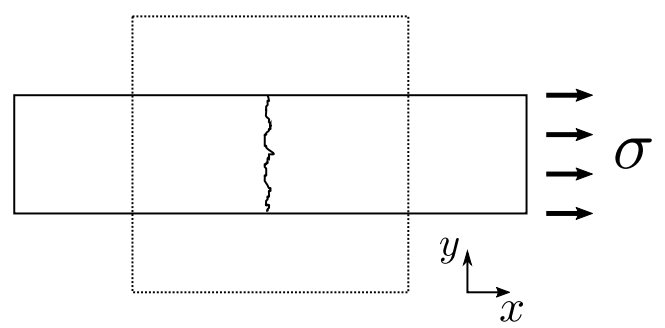

Figure 25: 2D uniaxial tension along $x$ axis

illustrated with the example of uniaxial tension along the $x$ axis of figure 25 . The stress tensor can be written:

$$
[\boldsymbol{\sigma}]=\left[\begin{array}{lll}
\sigma & 0 & 0 \\
0 & 0 & 0 \\
0 & 0 & 0
\end{array}\right]
$$

The strain response is:

$$
[\boldsymbol{\epsilon}]=\frac{\sigma}{E}\left[\begin{array}{ccc}
1 & 0 & 0 \\
0 & -\nu & 0 \\
0 & 0 & -\nu
\end{array}\right]
$$

Whether it is on the example of a $1 \mathrm{D}$ bar as studied in section 2 or on this $2 \mathrm{D}$ example, with the CZM a crack is expected to appear when $\sigma$ reaches the critical stress value $\sigma_{c}$ :

$$
\sigma=\sigma_{c}
$$

With the TLS approach (V1 or V2), damage starts to appear when the energy release rate $Y$ reaches the critical value $Y_{\mathrm{c}}$; in $1 \mathrm{D}$ it can be expressed in order to have equivalence with a CZM as a function of $\sigma_{c}$ with equations (23) and (B.1):

$$
Y_{\mathrm{c}}^{1 D}=\frac{1}{2} \frac{\sigma_{c}^{2}}{E}
$$

This value of $Y c^{1 D}$ ensures that, in $1 \mathrm{D}$, cohesive crack initiation with the CZM or damage initiation with the TLS model both start when the stress in the bar reaches the value $\sigma_{c}$. If we compute $Y$ for the uniaxial tension example of figure (25) for $\sigma=\sigma_{c}$, using the expression of $Y$ given by equation (32) (with $\beta=0$ or $\beta=1$ ) and the expression (69) of $\boldsymbol{\epsilon}$, we obtain:

$$
Y\left(\sigma=\sigma_{c}\right)=\gamma(\nu) \frac{1}{2} \frac{\sigma_{c}^{2}}{E}
$$

where $\gamma(\nu) \neq 1$ is a function of $\nu$ which depends on whether the uniaxial tension test is studied under plane stress, plane strain or 3D conditions. Equation (72) implies that damage will start to appear for a value of $\sigma$ which is different from $\sigma_{c}$. Therefore, the cohesive, TLSV1 and TLSV2 models are not fully equivalent in $2 \mathrm{D}$, which can explain why the values of the dissipated energy given in table 5 shows some differences between the CZM and the TLSV2 model (for the TLSV1, it was shown that numerical issues are another source of error).

Figure 26 shows, for a mesh element size of $h_{e}=\ell_{c} / 5$, the influence of the critical value $\phi^{*}$ in the TLSV2. The value of $\phi^{*}$ does not have a significant impact on the results. Only for the value of $\phi^{*}=0.9 \ell_{\mathrm{c}}$, the results obtained seem to dissipate a bit more energy than for the other values of $\phi^{*}$. We have seen previously that the TLSV1 dissipates more energy than the TLSV2, and for $\phi^{*} \rightarrow \ell_{\mathrm{c}}$, 

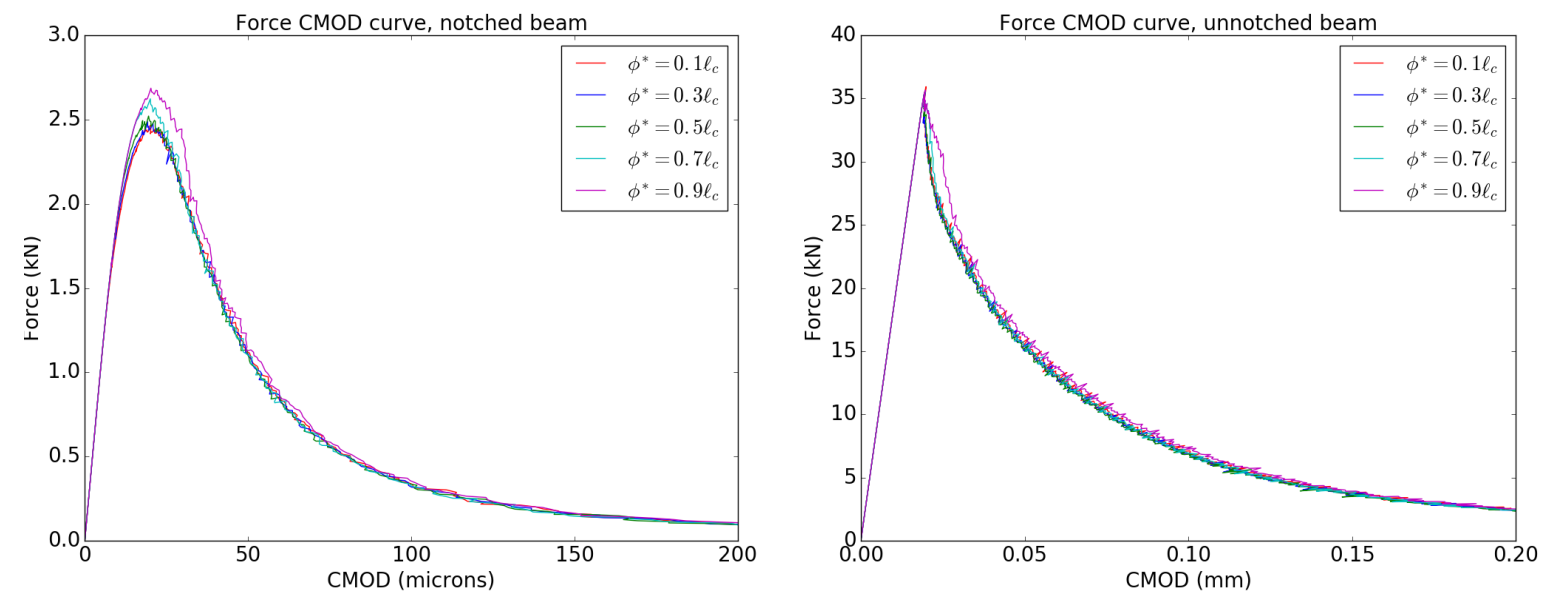

Figure 26: Three-points bending tests on notched (left) and unnotched (right) beam: force CMOD curves, TLSV2, influence of the critical value $\phi^{*}$.

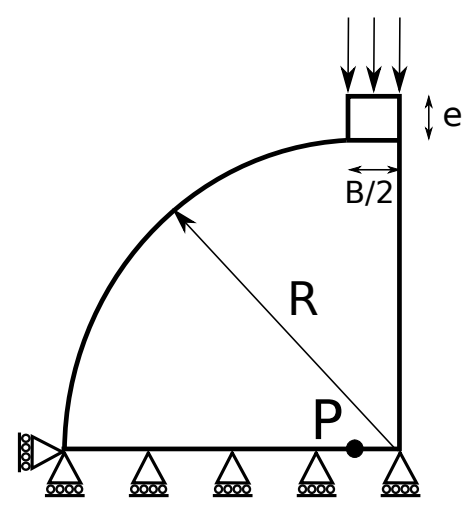

Figure 27: Splitting test: geometry and boundary conditions.

the bulk damage variable $D$ tends to 1 , therefore the numerical problems evoked at the end of section 5.2 may start to reappear, and the TLSV2 is expected to tend to the TLSV1.

\subsection{Splitting test}

The objective of this example is to show the ability of the TLSV2 to handle stress triaxiality. The radius of the specimen is $R=400 \mathrm{~mm}$, the width of the loading bearing strips is $B=12 \mathrm{~mm}$ and their thickness is $e=6 \mathrm{~m}$ (see figure 27). The CMOD is defined as twice (because of the symmetric boundary conditions) the horizontal displacement of a point $\mathrm{P}$ located at coordinates $(-B / 2,0)$, assuming that the center of the cylinder is located at coordinates $(0,0)$. The characteristic length is $\ell_{\mathrm{c}}=5 \mathrm{~mm}$, and for the TLSV2 model, the critical value is $\phi^{*}=0.5 \ell_{\mathrm{c}}$. The simulations are performed under plane stress assumption, with a mesh element size of $h_{e}=\ell_{\mathrm{c}} / 5$ in the center part of the specimen, where the crack is expected to propagate. The material parameters of the gradual model are given in table 6 .

The bulk and cohesive damage at different computation steps are shown on figure 28. As observed by other authors [37], the crack initiates at about $2 R / 3$ from the center of the cylinder. Because of the compressive zone under the loading bearing strips, the crack propagates toward the center of the sample. Finally, the crack grows upward until it reaches the bearing strip. Note that while $\phi<\phi^{*}$ (figure 28 (a)), there is no cohesive damage (and therefore no displacement jump). Then $\phi$ increases until $\phi>\phi^{*}$, cohesive damage starts to appear (figure 28 (b) and (c)). Finally, when $\phi>\ell_{\mathrm{c}}$, the cohesive damage reaches the value 1 (figure $28(\mathrm{~d})$. The maximum value of the cohesive damage $d$ is 


\begin{tabular}{|l|c|c|}
\hline Elastic parameters & $E(\mathrm{MPa})$ & 37700 \\
& $\nu$ & 0,2 \\
\hline \multirow{4}{*}{ Gradual model parameters } & $\sigma_{c}(\mathrm{MPa})$ & 3,77 \\
& $\sigma_{k}(\mathrm{MPa})$ & 0,57 \\
& $w_{c}(\mathrm{~mm})$ & 0,04 \\
& $w_{1}(\mathrm{~mm})$ & 0,002 \\
\hline
\end{tabular}

Table 6: Splitting test: material parameters.

not exactly 1 , because the width of the damage band is slightly lower than $\ell_{\mathrm{c}}$ ).

Figure 29 shows, for the first step of a computation done with the pure CZM, the positive part of the $x x$ component of the strain field and the cohesive energy release rate $y$ which drives the evolution of the cohesive damage variable field (computed by solving the modified Lagrangian formulation, see equation (54)). The $x x$ component of the strain field is the quantity which is expected to govern the initiation and propagation of cracks, and as expected its maximum is about $2 R / 3$ from the center of the cylinder, where the damage initiates with the TLS approach (figure 28 (a)). On the contrary, $y$ reaches its maximum at the center of the cylinder, therefore the crack initiates at this point, and then propagates toward the top of the cylinder.

Figure 30 shows the force-CMOD curves obtained with the cohesive, the TLSV1 and the TLSV2 models, for a mesh where the element size is $h_{e}=\ell_{\mathrm{c}} / 5$. As expected, the CZM gives a very different behavior; in particular, the cohesive crack starts to open for a loading which is superior the one which is necessary for the damage to appear with the TLSV1 or V2 models.

\section{Discussion}

In this paper, a new method to combine damage mechanics and cohesive zone models has been proposed, thanks to the Thick Level Set approach to fracture. The new approach (TLSV2) is characterized by two damage variables, a bulk damage variable and a cohesive damage variable, both being functions of the same level set field. In the TLSV2, the damage in the bulk is restricted to a value strictly inferior to 1, preventing numerical issues which may occur with classical damage models when damage tends to 1, like infinite strains for instance. A 1D equivalence was established with the first version of the TLS (TLSV1) with only bulk damage and with the CZM.

The TLSV1 and V2, as well as the cohesive zone model, were compared on several examples, in 1D and in 2D. It was shown that the convergence speed of the TLSV2 is much faster than the one of the TLSV1, with results which almost do not depend on the size of the elements of the mesh, proving that the aforementioned numerical issues of the TLSV1 are solved by the TLSV2. Even if a few differences can be observed between both (especially on the dissipated energy), the TLSV2 gives results which are very close to the cohesive zone model. It has also been shown that the TLSV2 can handle stress triaxiality, which is not the the case of the pure CZM model. However, one of the essential aspects of the TLSV2 is the use of the skeleton of the level set field. Assuming that it coincides with the axis of symmetry in symmetric examples allowed to get some first interesting results, but the localization of the skeleton in any situation is necessary in order to fully illustrate the potential of the TLSV2. Indeed, contrary to existing approaches where the cohesive crack location needs to be determined, it should be automatically given here as the skeleton of the level set, that is to say the surface where its gradient is discontinuous. Also, compared to a pure CZM model, the bulk part of the TLSV2 is expected to provide a natural propagation criterion, as well as the possibility for cracks to branch and coalesce. Another point is the capacity of the TLSV2 to apply interfacial behaviors (like frictional contact for instance) on the crack lips. From a numerical point of view, this is not simple with the TLSV1 for at least two reasons. The first one is that the crack is represented by two surfaces (the two sides of the iso- $\ell_{c}$ ), which requires to relate each point of a crack lip with its counterpart on the other lip. The second reason is that with the TLSV1, the bulk material is very soft around the macro-crack because of the bulk damage variable tending to 1 . These two problems should disappear with the TLSV2, since the geometry of the macro crack is a surface corresponding to the skeleton, and the bulk damage 


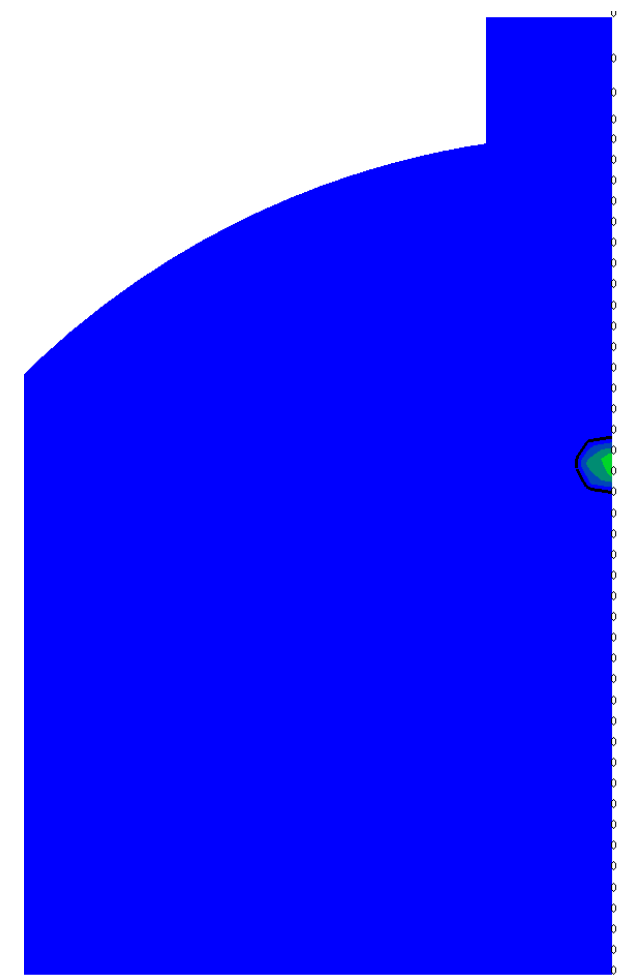

(a)

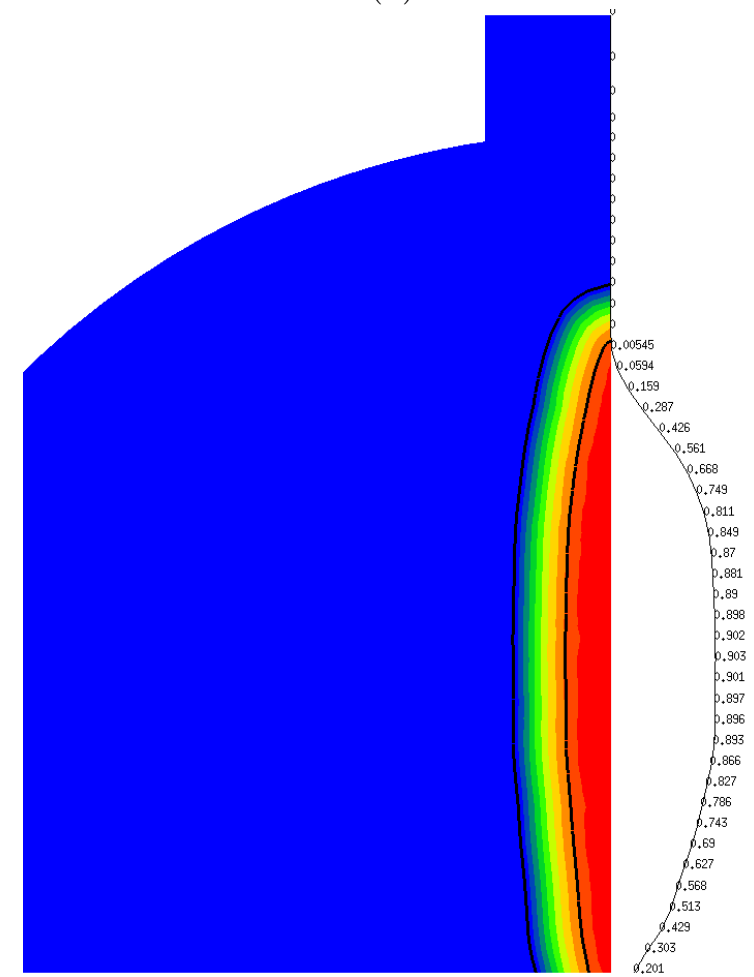

(c)

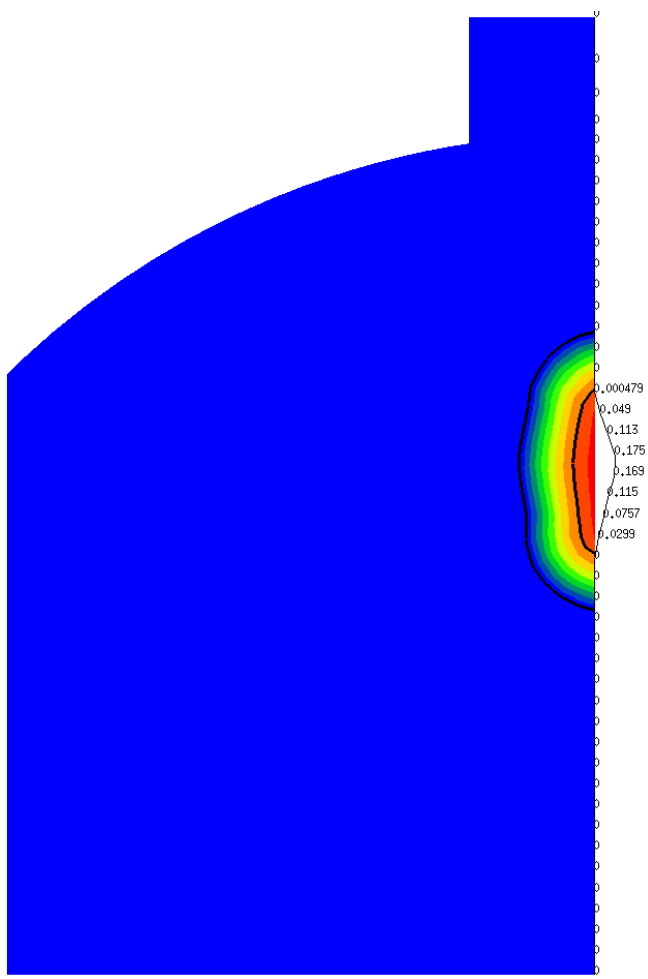

(b)

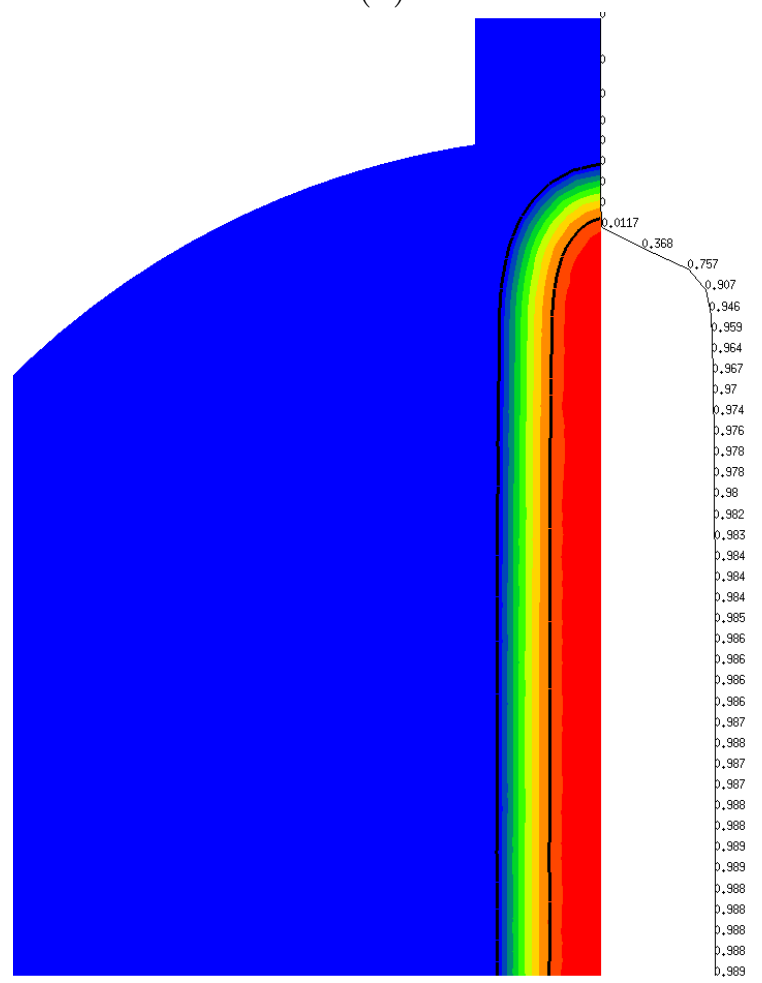

(d)

Damage

0.458

0.917

Figure 28: Splitting test, TLSV2 model: bulk damage field (color map) and cohesive damage (black line) at different computation steps. The black thick lines are the iso-zero and iso- $\phi^{*}$ of the level set field. 


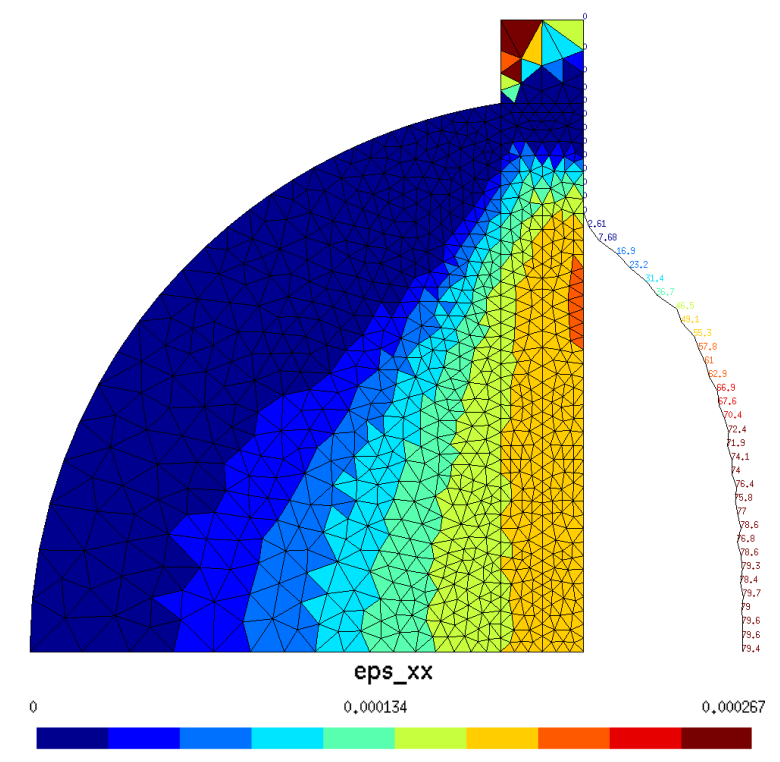

Figure 29: Splitting test, CZM: $x x$ strain field component (color map: only the positive values are plotted for clarity) and cohesive energy release rate $y$ field (line on the right) for the first computation step.

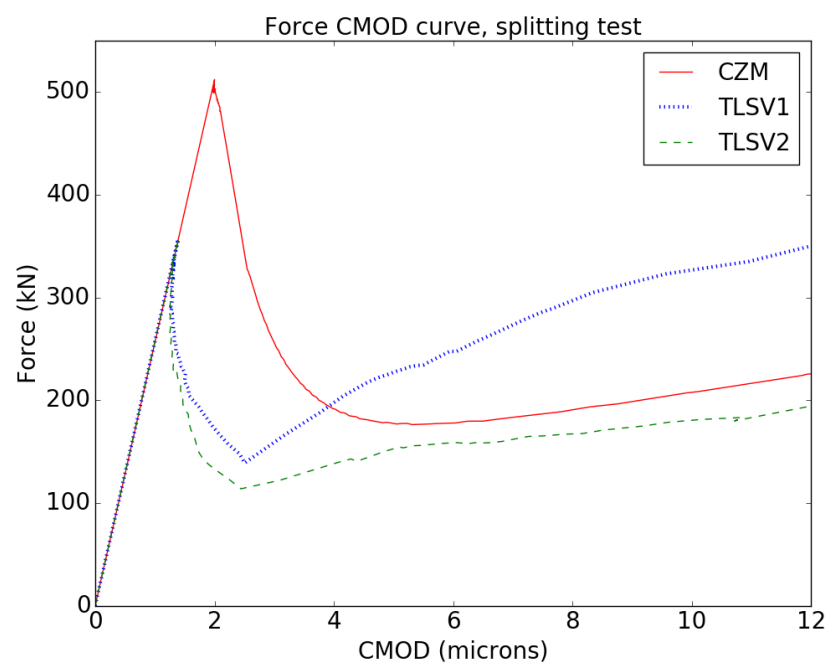

Figure 30: Splitting test: force CMOD curves, comparison between the CZM, the TLSV1 and the TLSV2 models. 
variable strictly inferior to 1 . These aspects will be illustrated in a forthcoming contribution, where we will also deal with non symmetric crack growth for which the skeleton extraction and enrichment will be needed. Finally, the parameters of the TLSV2 were chosen using a 1D equivalence with the TLSV1 of the CZM model. However a more general method to determine them directly, without using another model as a starting point, should be investigated.

Note that in this paper we focused on softening only damage models, but damage models exhibiting first a hardening behavior may also be analyzed with the TLS [29, 30].

\section{Acknowledgment}

The support of the ERC Grant XLS n ${ }^{\circ} 291102$ is gratefully acknowledged. We also would like to thank Alexis Salzman and Nicolas Chevaugeon for the many discussions about the TLS V2.

\section{Appendix A. From TLSV1 to CZM [32]}

This section is a reminder from [32], where a softening function $H$ is deduced in order to have a global behavior of the TLSV1 model equivalent to a bilinear CZM (cf. figure 13). In order to simplify the notations, the subscript "1" indicating the TLSV1 quantities are removed in this subsection.

The following dimensionless quantities are introduced:

$$
\hat{w}_{1}=w_{1} / w_{c}, \hat{w}_{k}=w_{k} / w_{c}, \hat{\sigma}_{k}=\sigma_{k} / \sigma_{c}, \lambda=\frac{2 \sigma_{c} \ell_{c}}{E w_{c}}
$$

The function $\tilde{H}(D)$, defined as

$$
\tilde{H}(D)=\int_{0}^{D} H(D) \mathrm{d} D
$$

is given, for the parabolic damage profile (17), by:

$$
\tilde{H}(D)=\frac{D}{1-D} \frac{1}{(1+\lambda C I(D))^{2}}
$$

with $C$ a constant depending on the value of $\widehat{\phi}=\phi / \ell_{\mathrm{c}}$ :

$$
C=\left\{\begin{array}{lll}
\lambda / \hat{w}_{1} & \text { if } \hat{\phi}<\widehat{\phi}_{k} \\
\frac{\lambda \hat{\sigma}_{k}}{1-\hat{w}_{k}} & \text { if } \hat{\phi}>\widehat{\phi}_{k}
\end{array}\right.
$$

where

$$
\widehat{\phi}_{k}=\frac{a}{2}(\sqrt{1+4 / a}-1)
$$

and

$$
I(D)=\frac{2-D}{\sqrt{1-D}}-2
$$

The critical value $\widehat{\phi}_{k}$ corresponds to the kink point in the traction-separation law $t=f(w)$. The primitive $\tilde{H}$ of the hardening function is continuous, but not its derivative, which explains the discontinuity of $H$ (see figure 14). We can also write $\tilde{H}$ as a function of $\widehat{\phi}$ :

$$
\tilde{H}(\widehat{\phi})=B \frac{2 \widehat{\phi}-\widehat{\phi}^{2}}{\left(1-\widehat{\phi}+\lambda C \widehat{\phi}^{2}\right)^{2}}
$$

with $B$ another constant depending on the value of $\widehat{\phi}$.

$$
B=\left\{\begin{array}{cl}
1 & \text { if } \widehat{\phi}<\widehat{\phi}_{k} \\
\left(\frac{\hat{\sigma}_{k}}{1-\hat{w}_{k}}\right)^{2} & \text { if } \hat{\phi}>\widehat{\phi}_{k}
\end{array}\right.
$$




\section{Appendix B. Cohesive zone model}

Cohesive zone models are usually given by a traction-separation relation $t=f(w)$. In order to fully determine a pure CZM using the formalism introduced in section 2.1, one needs to compute $y_{\mathrm{c}}$ and $h$ (In order to simplify the notations, the subscript "1" indicating the CZM quantities are removed in this subsection). Having $d=0$ corresponds to $t=\sigma_{c}$ and $w=0$, so by substituting $\frac{w}{d}$ in the expression of $t$ in (2), and using the fact that $y=y_{\mathrm{c}} h(0)=y_{\mathrm{c}}$ when $d$ starts to grow, the expression of $y_{\mathrm{c}}$ is:

$$
y_{\mathrm{c}}=\frac{\sigma_{c}^{2}}{2 k}
$$

When $\dot{d}>0$, the criterion in (3) becomes an equality, leading to:

$$
y_{\mathrm{c}} h(d)=y=\frac{1}{2} k\left(\frac{w}{d}\right)^{2}
$$

Using the first equation of the state equations (2) and the relation $t=f(w), w$ can be expressed as a function of $d$, leading to the expression of $h(d)$. It can be noticed that whatever the value of $k$ in equation (B.2), the same traction-separation law $t=f(w)$ is recovered.

For instance, in the case of the bilinear law [36] depicted in figure 13, and written:

$$
t=a-b w
$$

where

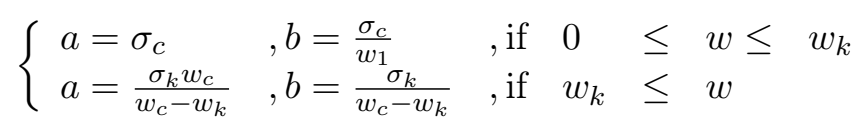

the expression of $y_{\mathrm{c}} h(d)$ is:

$$
y_{\mathrm{c}} h(d)=\frac{k}{2}\left(\frac{a}{k(1-d)+b d}\right)^{2}
$$

\section{Appendix C. From TLSV1 to TLSV2}

To determine the cohesive damage profile $d\left(\phi_{s}\right)$, one has to start from the stiffness equivalence (21). Then, using the expression of $k$ given by (19) we obtain:

$$
\left.\frac{d}{1-d}\right|_{\phi=\phi_{s}}=\frac{1}{\ell_{\mathrm{c}}}\left(\int_{\phi^{*}}^{\phi_{s}} \frac{1}{1-D_{1}}-\frac{1}{1-D} \mathrm{~d} x\right)
$$

For $\phi>\phi^{*}$ we choose $D(\phi)$ such that:

$$
\frac{1}{1-D(\phi)}=S\left(\phi^{*}\right)+S^{\prime}\left(\phi^{*}\right)\left(\phi-\phi^{*}\right)
$$

where

$$
S(\phi)=\frac{1}{1-D_{1}(\phi)}
$$

The use of this function $S$ allows to compute easily the integrals in (C.1), as well as to ensure the $\mathcal{C}^{1}$ continuity of $D(\phi)$ at $\phi=\phi^{*}$. From (C.1) we can finally deduce $d\left(\phi_{s}\right)$ :

$$
d\left(\phi_{s}\right)=\frac{R\left(\phi_{s}\right)}{1+R\left(\phi_{s}\right)}
$$

with

$$
R(\phi)=\frac{1}{\sqrt{1-D_{1}(\phi)}}-\frac{1}{\sqrt{1-D^{*}}}-\frac{1}{\ell_{\mathrm{c}}}\left(S\left(\phi^{*}\right)\left(\phi-\phi^{*}\right)+\frac{S^{\prime}\left(\phi^{*}\right)}{2}\left(\phi-\phi^{*}\right)^{2}\right)
$$


From equation (22) and expression (20) for $y_{\mathrm{c}}$, the expression of $h(d)$ is obtained:

$$
h\left(d\left(\phi_{s}\right)\right)=\frac{\tilde{H}\left(D_{1}\left(\phi_{s}\right)\right)-\tilde{H}\left(D\left(\phi_{s}\right)\right)}{\ell_{\mathrm{c}} d^{\prime}\left(\phi_{s}\right)}
$$

where $\tilde{H}$ is given by equation (A.2).

\section{Appendix D. From CZM to TLSV2}

In the framework of the equivalence between CZM and TLSV2, the softening function $H$ is obtained from the dissipation equivalence (24), which reduces, for $\phi_{s}<\phi^{*}$, to:

$$
2 \int_{0}^{\phi_{s}} Y_{\mathrm{c}} H(D) D^{\prime} \mathrm{d} x=\left.y_{c 1} h_{1}\left(d_{1}\right) d_{1}^{\prime}\right|_{\phi=\phi_{s}}
$$

with $d_{1}$ given by:

$$
2 \int_{0}^{\phi_{s}} \frac{1}{E} \frac{D}{1-D} \mathrm{~d} x=\left.\frac{1}{k_{1}} \frac{d_{1}}{1-d_{1}}\right|_{\phi=\phi_{s}}
$$

It can be noticed that for $\phi<\phi^{*}$, the cohesive part of the TLSV2 is not active. Therefore, the function $H$ that will be obtained from equations (D.1) and (D.2) will be the same as the one obtained by [32], where a TLSV1 model was derived from a CZM.

The function $h$ is then obtained from equations (24) and (25), for $\phi_{s}>\phi^{*}$. Equation (24) has two unknowns $h$ and $H$, which gives a certain freedom. We can either choose an expression for $h$ and have $H$ be determined by (24), or choose the expression of $H$ and have the expression of $h$ imposed by (24). The former, where we choose for $H(D)$ with $D>D\left(\phi^{*}\right)$ the same expression than for $D<D\left(\phi^{*}\right)$, seems to be the easiest and most reasonable choice, as it ensures the $\mathcal{C}^{1}$ continuity of $H(D(\phi))$ at $\phi_{s}=\phi^{*}$. However, as all the computations done in this paper where performed with a TLSV2 model derived from a TLSV1 model, the best choice to determine $H$ and $h$ for $\phi_{s}>\phi^{*}$ could still be discussed.

\section{Appendix E. Explicit algorithm}

We present in this section a summary of the explicit algorithm developed in [30] used to solve a problem with the Thick Level Set approach, whether it is the TLSV1 or the TLSV2.

\section{General flowchart}

First of all, the loading $\boldsymbol{F}$ (which can be prescribed forces or Dirichlet boundary conditions) is written as:

$$
\boldsymbol{F}=\mu \boldsymbol{F}_{\text {ref }}
$$

where $\boldsymbol{F}_{\text {ref }}$ is an arbitrary loading and $\mu$ is a load factor, which is supposed to be an unknown of the problem. Therefore, the following quantities need to be determined at each computation step:

- The displacement field $\boldsymbol{u}$

- The level set field $\phi$

- The load factor $\mu$

These quantities are computed using and explicit algorithm made of three steps; assuming that $\left(\boldsymbol{u}^{n}, \phi^{n}, \mu^{n}\right)$ at computation step $n$ are known:

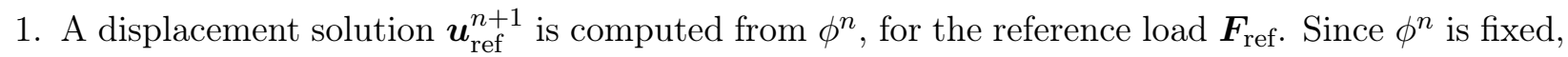
the problem is linear with respect to the displacement unknown, unless the asymmetric potential (29) is used; in that case a Newton-Raphson algorithm is used.

2. The load factor $\mu^{n+1}$ is computed (see equation (E.8)).

3. Finally, the increment of level-set field $\Delta \phi$ is computed from $\boldsymbol{u}_{\mathrm{ref}}^{n+1}$ and $\mu^{n+1}$, and the level-set field is updated: $\phi^{n+1}=\phi^{n}+\Delta \phi$. 


\section{Level set update}

Let $f_{I}^{n}$ denote the evolution criterion for a given time step $n$ and for a given mode $I \in M$ :

$$
f_{I}^{n}=\left(\mu^{n}\right)^{2} a_{I}^{n}-b_{I}^{n}
$$

with

$$
\begin{cases}a_{I}^{n}=\bar{Y}_{\mathrm{ref}, I}^{n}, & \forall I \in M \\ b_{I}^{n}=Y_{\mathrm{c}} \bar{H}_{I}^{n}, & \forall I \in M\end{cases}
$$

for the TLSV1 and

$$
\left\{\begin{array}{l}
a_{I}^{n}=g_{\mathrm{ref}, I}^{n}, \quad \forall I \in M \\
b_{I}^{n}=g_{c, I}^{n}, \quad \forall I \in M
\end{array}\right.
$$

for the TLSV2.

The load factor $\mu^{n}$ at step $n$ is computed in order to have:

$$
\max _{I \in M}\left(f_{I}^{n}\right)=0
$$

Which gives:

$$
\mu^{n}=\min _{I \in M} \sqrt{\frac{b_{I}^{n}}{a_{I}^{n}}}
$$

The increment of $\phi$ at each mode $\Delta \phi_{I}$ is computed using a prediction $f_{I}^{\text {pred }}$ of the criterion:

$$
\left\{\begin{aligned}
f_{I}^{\text {pred }} & \leq 0, \quad \forall I \in M \\
\Delta \phi_{I} & \geq 0, \quad \forall I \in M \\
f_{I}^{\text {pred }} \Delta \phi_{I} & =0, \quad \forall I \in M
\end{aligned}\right.
$$

where $\Delta \phi_{I}$ is computed such that

$$
\max _{I \in M} \Delta \phi_{I}=\Delta \phi_{\max }
$$

The prediction of the evolution criterion is computed as

$$
f_{I}^{\text {pred }}=f_{I}^{n}+\alpha_{I}^{n} \Delta \mu^{\text {pred }}-\beta_{I}^{n} \Delta \phi_{I}
$$

where

$$
\left\{\begin{array}{l}
\alpha_{I}^{n}=2 \mu^{n} \bar{Y}_{\text {ref,I }}^{n}, \quad \forall I \in M \\
\beta_{I}^{n}=\frac{\int_{\Omega} Y_{c} H^{\prime}\left(D_{1}\right) D_{1}^{\prime 2} M_{I} \mathrm{~d} \Omega}{\int_{\Omega} D_{1}^{\prime} M_{I} \mathrm{~d} \Omega}, \forall I \in M
\end{array}\right.
$$

for the TLSV1 and

$$
\left\{\begin{array}{l}
\alpha_{I}^{n}=2 \mu^{n} g_{\mathrm{ref}, I}^{n}, \quad \forall I \in M \\
\beta_{I}^{n}=\int_{\Omega} Y_{c} H^{\prime}(D) D^{\prime 2} M_{I} \mathrm{~d} \Omega+\int_{\Gamma_{s}} \frac{1}{2} y_{\mathrm{c}} h^{\prime}(d) d^{\prime 2} M_{I} \mathrm{~d} \Gamma, \quad \forall I \in M
\end{array}\right.
$$

for the TLSV2.

The value of $\Delta \mu^{\text {pred }}$ which verifies (E.12) is

$$
\Delta \mu^{\mathrm{pred}}=\min _{I \in M} \frac{\beta_{I}^{n} \Delta \phi_{\max }-f_{I}^{n}}{\alpha_{I}^{n}}
$$

Finally $\Delta \phi_{I}$ is computed as

$$
\Delta \phi_{I}=\max \left(0, \frac{f_{I}^{n}+\Delta \mu^{\text {pred }} \alpha_{I}^{n}}{\beta_{I}^{n}}\right), \forall I \in M
$$

In all the computations presented in this paper, a value of $\Delta \phi_{\max }=0.25 \mathrm{~h}$ is chosen (h being the size of the elements of the mesh). 


\section{Spatial diffusion}

In order to reduce spatial oscillations of the non-local fields, the variational formulation (40) used to compute $\bar{X}$ is modified by adding a numerical diffusion term to the left-hand side:

$$
\int_{\Gamma_{0}} \overline{X X}^{*} \mathrm{~d} \Omega+\int_{\Gamma_{0}}(\beta h)^{2} \nabla \bar{X} \cdot \nabla \bar{X}^{*} \mathrm{~d} \Gamma=\int_{\Omega} X D^{\prime} \bar{X}^{*} \mathrm{~d} \Omega+\int_{\Gamma_{s}} x d^{\prime} \bar{X}^{*} \mathrm{~d} \Gamma, \forall \bar{X}^{*} \in \overline{\mathscr{Y}}
$$

where $\beta$ is a user defined parameter and $h$ the characteristic finite element size. We obtained the new matricial system which needs to be solved to compute the coefficients $\bar{X}_{I}$ :

$$
[\boldsymbol{K}]\{\overline{\boldsymbol{X}}\}=[\boldsymbol{M}]\{\boldsymbol{A}\}
$$

where

$$
[\boldsymbol{K}]=[\boldsymbol{M}]\left[\boldsymbol{K}^{N}\right][\boldsymbol{M}]^{\top}
$$

and

$$
K_{i j}^{N}=\int_{\Omega} L_{I} D^{\prime} L_{j} \mathrm{~d} \Omega+\int_{\Gamma_{0}}(\beta h)^{2} \nabla L_{I} \cdot \nabla L_{j} \mathrm{~d} \Gamma, \quad \forall i, j \in N
$$

\section{Appendix F. Explicit algorithm; adaptation to the CZM}

The explicit algorithm which was presented in the precedent section can also be used to solve a CZM problem, with the following modifications:

- In CZM, the damage variable $d$ (by ignoring the subscript "1" indicating the CZM quantities in this subsection) does not depend on an auxiliary level set field, therefore the unknown $\phi$ in the algorithm of section Appendix F must be replaced by $d$

- In equation (E.2), the expressions of $a_{I}$ and $b_{I}$ are:

$$
\begin{cases}a_{I}^{n}=y_{\mathrm{ref}, I}^{n}, & \forall I \in M \\ b_{I}^{n}=y_{\mathrm{c}} h_{I}^{n}, & \forall I \in M\end{cases}
$$

Note that this arises the question of the nature of $M$ in the CZM case. In the TLS framework, $M$ was the set of propagation modes, related to the concept of non-locality, which does not exist in our CZM approach. Therefore, for the CZM the set $M$ is simply a set of nodes corresponding to a discretization of the crack path. The notation $x_{I}^{n}$ corresponds to the quantity $x$ at computation step $n$, evaluated at node $I$.

- In equation (E.13), the expression of $\alpha_{I}^{n}$ and $\beta_{I}^{n}$ are:

$$
\left\{\begin{array}{l}
\alpha_{I}^{n}=2 \mu^{n} y_{\mathrm{ref}, I}^{n}, \quad \forall I \in M \\
\beta_{I}^{n}=y_{\mathrm{c}} h_{I}^{\prime n}, \quad \forall I \in M
\end{array}\right.
$$

- The user defined parameter $\Delta \phi_{\max }$, maximum increment of $\phi$, becomes $\Delta d_{\max }$, a maximum increment of $d$

Numerical diffusion is introduced directly into the expression of $f_{I}^{\text {pred }}$ through a numerical parameter $\alpha$;

$$
f_{I}^{\mathrm{pred}}=f_{I}^{n}+\alpha_{I}^{n} \Delta \mu^{\mathrm{pred}}-\left(1+\frac{\alpha h_{e} \mu^{1}}{\Delta \mu^{\mathrm{pred}}}\right) \beta_{I}^{n} \Delta d_{I}
$$

where $h_{e}$ is the finite element size and $\mu^{1}$ is the load factor for the first computation step. Introducing it in equation (F.5) allows to obtain an algorithm independent from the reference loading. The value of $\Delta \mu^{\text {pred }}$ which verifies (E.12) is now solution of a second order polynomial, which can be written as:

$$
\Delta \mu^{\text {pred }}=\min _{I \in M} F_{I}
$$


where

$$
F_{I}=\frac{1}{2} \frac{\left(\beta_{I}^{n} \Delta d_{\max }-f_{I}^{n}\right)}{\alpha_{I}^{n}}\left(1+\sqrt{1+\frac{4 \alpha h_{e} \alpha_{I}^{n} \beta_{I}^{n} \Delta d_{\max }}{\left(f_{I}^{n}-\beta_{I}^{n} \Delta d_{\max }\right)^{2}}}\right)
$$

Finally $\Delta \phi_{I}$ is computed as

$$
\Delta d_{I}=\max \left(0, \frac{f_{I}^{n}+\Delta \mu^{\mathrm{pred}} \alpha_{I}^{n}}{\left(1+\frac{\alpha h_{e} \mu^{1}}{\Delta \mu^{\text {pred }}}\right) \beta_{I}^{n}}\right), \forall I \in M
$$

\section{Appendix G. Dissipation}

With the TLSV2 approach, the power dissipated is equal to:

$$
\begin{aligned}
\delta W_{d} & =\int_{\Gamma_{t}} \boldsymbol{F} \cdot \dot{\boldsymbol{u}} \mathrm{d} \Gamma-\frac{\mathrm{d}}{\mathrm{d} t} W(\boldsymbol{u}, \phi) \\
& =\int_{\Omega} \boldsymbol{\sigma}: \boldsymbol{\epsilon}(\dot{\boldsymbol{u}}) \mathrm{d} \Omega+\int_{\Gamma_{\mathrm{s}}(\phi)} \boldsymbol{t} \cdot[\dot{\boldsymbol{u}}] \mathrm{d} \Gamma-\frac{\mathrm{d}}{\mathrm{d} t} W(\boldsymbol{u}, \phi) \\
& =\int_{\Omega}\left(\boldsymbol{\sigma}-\Psi_{,} \boldsymbol{\epsilon}\right): \boldsymbol{\epsilon}(\dot{\boldsymbol{u}})-\Psi_{, D} \dot{D} \mathrm{~d} \Omega+\int_{\Gamma_{\mathrm{s}}(\phi)}\left(\boldsymbol{t}-\varphi_{,}, \llbracket \boldsymbol{u} \rrbracket\right) \cdot[\dot{\boldsymbol{u}}]-\varphi_{, d} \dot{d} \mathrm{~d} \Gamma \\
& =\int_{\Omega} Y_{c} H(D) \dot{D} \mathrm{~d} \Omega+\int_{\Gamma_{\mathrm{s}}(\phi)} y_{c} h(d) \dot{d} \mathrm{~d} \Gamma
\end{aligned}
$$

The last equality is valid if the model is so called associated (the stress are the derivative of the energy and the derivative of the energy with respect to damage drives the damage evolution). The total work dissipated during a period $[0 ; t]$ is:

$$
\begin{aligned}
W_{d} & =\int_{0}^{t} \delta W_{d} \\
& =\int_{\Omega}\left(\int_{0}^{t} Y_{c} H(D) \dot{D} \mathrm{~d} t\right) \mathrm{d} \Omega+\int_{\Gamma_{\mathrm{s}}(\phi)}\left(\int_{0}^{t} y_{c} h(d) \dot{d} \mathrm{~d} t\right) \mathrm{d} \Gamma \\
& =\int_{\Omega} Y_{c} \tilde{H}(D) \mathrm{d} \Omega+\int_{\Gamma_{\mathrm{s}}(\phi)} y_{c} \tilde{h}(d) \mathrm{d} \Gamma
\end{aligned}
$$

We consider the particular case of a straight crack of figure G.31, and compute the energy dissipated between two positions where the macro-crack has already appeared. Then

$$
\begin{aligned}
W_{d, 1-2} & =\int_{\Omega_{c 2} \backslash \Omega_{c 1}} Y_{c} \tilde{H}(D) \mathrm{d} \Omega+\int_{\Gamma_{s 2}(\phi) \backslash \Gamma_{s 1}(\phi)} y_{c} \tilde{h}(d) \mathrm{d} \Gamma \\
& =\int_{-\ell_{c}}^{\ell_{c}} \int_{y_{1}}^{y_{2}} Y_{c} \tilde{H}(D) \mathrm{d} y \mathrm{~d} x+\int_{y_{1}}^{y_{2}} y_{c} \tilde{h}(d) \mathrm{d} y \\
& =2 Y_{c}\left(y_{2}-y_{1}\right) \int_{0}^{\ell_{c}} \tilde{H}(D) \mathrm{d} x+y_{c}\left(y_{2}-y_{1}\right) \tilde{h}(1) \\
& =2 Y_{c} \ell_{c}\left(y_{2}-y_{1}\right)\left(\int_{0}^{D\left(\ell_{c}\right)} \frac{\tilde{H}(D)}{G(D)} \mathrm{d} D+\tilde{h}(1)\right)
\end{aligned}
$$

where

$$
G(D)=D^{\prime}\left(D^{-1}(D)\right)
$$

Applying the same reasoning for the TLSV1 approach, the total energy dissipated is: 


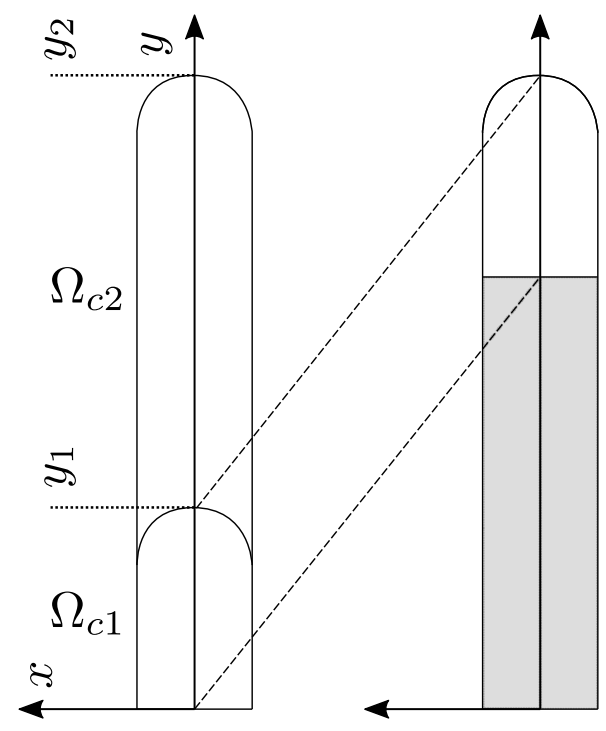

Figure G.31: Computation of the energy dissipated by the propagation of a straight damaged zone, between two positions $y_{1}$ and $y_{2}$

$$
W_{d, 1-2}=2 Y_{c} \ell_{c}\left(y_{2}-y_{1}\right) \int_{0}^{1} \frac{\tilde{H}\left(D_{1}\right)}{G\left(D_{1}\right)} \mathrm{d} D_{1}
$$

By taking equation (22) and integrating it over time, we obtain the equality between the TLSV1 dissipation (G.14) and the TLSV2 dissipation (G.11).

\section{References}

[1] Grigory Isaakovich Baranblatt. The mathematical theory of equilibrium cracks formed by brittle fracture. Zh. Prkl. Mekh. Tekh. Fiz, (4):3-56, 1961.

[2] Zdeněk P. Bažant. Instability, Ductility and Size-effect in Strain-Softening Concrete. Journal Of The Engineering Mechanics Division, 102(2):331-343, 1976.

[3] Paul Émile Bernard, Nicolas Moës, and Nicolas Chevaugeon. Damage growth modeling using the Thick Level Set (TLS) approach: Efficient discretization for quasi-static loadings. Computer Methods in Applied Mechanics and Engineering, 233-236:11-27, aug 2012.

[4] Fabien Cazes, Michel Coret, and Alain Combescure. A two-field modified Lagrangian formulation for robust simulations of extrinsic cohesive zone models. Computational Mechanics, 51:865-884, 2013.

[5] Fabien Cazes, Michel Coret, Alain Combescure, and Anthony Gravouil. A thermodynamic method for the construction of a cohesive law from a nonlocal damage model. International Journal of Solids and Structures, 46(6):1476-1490, 2009.

[6] Fabien Cazes and Nicolas Moës. Comparison of a phase-field model and of a thick level set model for brittle and quasi-brittle fracture. International Journal for Numerical Methods in Engineering, 103(February):114-143, 2015.

[7] Jean-Louis Chaboche and Jean Lemaitre. Mécanique des matériaux solides. Paris, dunod edition, 1988. 
[8] René Chambon, Denis Caillerie, and Nada El Hassan. One-dimensional localisation studied with a second grade model. European Journal of Mechanics - A/Solids, 17(4):637-656, 1998.

[9] Claudia Comi, Stefano Mariani, and Umberto Perego. An extended FE strategy for transition from continuum damage to mode I cohesive crack propagation. International Journal for Numerical and Analytical Methods in Geomechanics, 31(2):213-238, 2007.

[10] Eugène Cosserat and François Cosserat. Théorie des corps déformables. Herman, Paris, 1909.

[11] Sam Cuvilliez. Passage d'un modèle d'endommagement continu régularisé à un modèle de fissuration cohésive dans le cadre de la rupture quasi-fragile. PhD thesis, Mines PariTech, 2012.

[12] Frédéric Dufour, Grégory Legrain, Gilles Pijaudier-cabot, and Antonio Huerta. Estimate of crack opening from a 2D continuum-based FE computation. International Journal for Numerical and Analytical Methods in Geomechanics, 36(16):1813-1830, 2012.

[13] Donald S. Dugdale. Yielding of steel sheets containing slits. Journal of the Mechanics and Physics of Solids, 8:100-104, 1960.

[14] Gilles A. Francfort and Jean-Jacques Marigo. Revisiting brittle fracture as an energy minimization problem. Journal of the Mechanics and Physics of Solids, 46(8):1319-1342, 1998.

[15] Bianca Giovanardi, Anna Scotti, and Luca Formaggia. A hybrid XFEM -Phase field (Xfield) method for crack propagation in brittle elastic materials. Computer Methods in Applied Mechanics and Engineering, 320:396-420, 2017.

[16] Cédric Giry, Frédéric Dufour, and Jacky Mazars. Stress-based nonlocal damage model. International Journal of Solids and Structures, 48:3431-3443, 2011.

[17] Arne Hillerborg, Modéer M., and Per-Erik Petersson. Analysis of crack formation and crack growth in concrete by means of fracture mechanics and finite elements. Cement and Concrete Research, 6:773-782, 1976.

[18] Christian G. Hoover and Zdeněk P. Bažant. Cohesive crack, size effect, crack band and workof-fracture models compared to comprehensive concrete fracture tests. International Journal of Fracture, 187(1):133-143, jan 2014.

[19] Lazar Kachanov. Rupture Time Under Creep Conditions. Otdelenie tekhnicheskich nauk, (8):2631, 1958.

[20] Alain Karma, David Kessler, and Herbert Levine. Phase-Field Model of Mode III Dynamic Fracture. Physical Review Letters, 87(4):045501, 2001.

[21] David Lasry and Ted Belytschko. Localization limiters in transient problems. International Journal of Solids and Structures, 24(6):581-597, 1988.

[22] Benoît Lé. Contribution à la simulation numérique des structures en béton armé utilisation: de fonctions de niveau pour la modélisation de la fissuration et des renforts. PhD thesis, Ecole Centrale de Nantes, 2016.

[23] Eric Lorentz, Sam Cuvilliez, and Kyrylo Kazymyrenko. Convergence of a gradient damage model toward a cohesive zone model. Comptes Rendus - Mecanique, 339(1):20-26, 2011.

[24] Eric Lorentz, Sam Cuvilliez, and Kyrylo Kazymyrenko. Modelling large crack propagation: from gradient damage to cohesive zone models. International Journal of Fracture, 178:85-95, 2012.

[25] Christian Miehe, Fabian Welschinger, and Martina Hofacker. Thermodynamically consistent phase-field models of fracture: Variational principles and multi-field FE implementations. International Journal for Numerical Methods in Engineering, 83(10):1273-1311, 2010. 
[26] Nicolas Moës and Ted Belytschko. Extended finite element method for cohesive crack growth. Engineering Fracture Mechanics, 69(7):813-833, may 2002.

[27] Nicolas Moës, John Dolbow, and Ted Belytschko. A finite element method for crack growth without remeshing. International Journal for Numerical Methods in Engineering, 150(February):131150, 1999 .

[28] Nicolas Moës, Claude Stolz, Paul Émile Bernard, and Nicolas Chevaugeon. A level set based model for damage growth : the thick level set approach. International Journal for Numerical Methods in Engineering, 86(3):358-380, 2011.

[29] Nicolas Moës, Claude Stolz, and Nicolas Chevaugeon. Coupling local and non-local damage evolutions with the Thick Level Set model. Advanced Modeling and Simulation in Engineering Sciences, 2(16):1-21, 2014.

[30] Kévin Moreau, Nicolas Moës, Nicolas Chevaugeon, and Alexis Salzman. Concurrent development of local and non-local damage with the Thick Level Set approach: implementation aspects and application to quasi-brittle failure. Computer Methods in Applied Mechanics and Engineering, pages $1-25,2017$.

[31] Kévin Moreau, Nicolas Moës, Didier Picart, and Laurent Stainier. Explicit dynamics with a non-local damage model using the thick level set approach. International Journal for Numerical Methods in Engineering, 102(3-4):808-838, 2015.

[32] Andrés Parrilla Gómez, Nicolas Moës, and Claude Stolz. Comparison between Thick Level Set (TLS) and cohesive zone models. Advanced Modeling and Simulation in Engineering Sciences, 2(1):1-22, 2015.

[33] Andrés Parrilla Gómez, Claude Stolz, Nicolas Moës, David Grégoire, and Gilles Pijaudier-cabot. On the capability of the Thick Level Set (TLS) damage model to fit experimental data of size and shape effects. Engineering Fracture Mechanics, 184:75-87, 2017.

[34] Ron H. J. Peerlings, W. A. Marcel Brekelmans, René de Borst, and Marc G. D. Geers. Gradientenhanced damage modelling of high-cycle fatigue. International Journal for Numerical Methods in Engineering, 49(12):1547-1569, 2000.

[35] Ron H. J. Peerlings, René de Borst, W. A. Marcel Brekelmans, and J. H. P. De Vree. Gradient Enhanced Damage for Quasi-Brittle Materials. International Journal for Numerical Methods in Engineering, 39(19):3391-3403, oct 1996.

[36] Per-Erik Petersson. Crack growth and development of fracture zones in plain concrete and similar materials. Division, Inst., 1981.

[37] Henry J. Petroski and Rasko P. Ojdrovic. The concrete cylinder: stress analysis and failure modes. International Journal of Fracture, 279:263-279, 1987.

[38] Gilles Pijaudier-Cabot and Zdeněk P. Bažant. Non local damage theory. Journal of Engineering Mechanics, 113(10):1512-1533, 1987.

[39] Joris J. C. Remmers, René de Borst, Clemens V Verhoosel, and A. Needleman. The cohesive band model : A cohesive surface formulation with stress triaxiality. International Journal of Fracture, 181(2):177-188, 2013.

[40] Alexis Salzman, Nicolas Moës, and Nicolas Chevaugeon. On use of the Thick Level Set method in $3 \mathrm{D}$ quasi-static crack simulation of quasi-brittle material. International Journal of Fracture, (Accepté), 2016.

[41] Mariana R. R. Seabra, Primož Šuštarič, Jose M. A. Cesar de Sa, and Tomaž Rodič. Damage driven crack initiation and propagation in ductile metals using XFEM. Computational Mechanics, 52:161-179, 2013. 
[42] Angelo Simone, Garth N. Wells, and Lambertus J. Sluys. From continuous to discontinuous failure in a gradient-enhanced continuum damage model. Computer Methods in Applied Mechanics and Engineering, 192(41-42):4581-4607, 2003.

[43] Claude Stolz and Nicolas Moës. A new model of damage: a moving thick layer approach. International Journal of Fracture, 174:49-60, 2012.

[44] Elena Tamayo-Mas and Antonio Rodríguez-Ferran. A new continuous-discontinuous damage model: Cohesive cracks via an accurate energy-transfer process. Theoretical and Applied Fracture Mechanics, 69:90-101, 2014.

[45] Garth N. Wells, Lambertus J. Sluys, and René de Borst. Simulating the propagation of displacement discontinuities in a regularized strain-softening medium. International Journal for Numerical Methods in Engineering, 53:1235-1256, 2002. 